PDXNet Portal

\title{
PDXNet Portal: Patient-Derived Xenograft model, data, workflow, and tool discovery
}

\author{
Running Title: \\ PDXNet Portal
}

\section{Authors:}

Soner Koc ${ }^{*}, 1$, Michael W. Lloyd ${ }^{*}, 2$, Jeffrey Grover ${ }^{1}$, Sara Seepo ${ }^{1}$, Sai Lakshmi Subramanian ${ }^{1}$, Manisha Ray ${ }^{1}$, Christian Frech ${ }^{1}$, John DiGiovanna ${ }^{1}$, Phillip Webster ${ }^{1}$, Steven Neuhauser ${ }^{2}$, Anuj Srivastava ${ }^{3}$, Xing Yi Woo ${ }^{3}$, Brian J. Sanderson ${ }^{3}$, Brian White ${ }^{3}$, Paul Lott ${ }^{4}$, PDXNet Consortium, Yvonne A. Evrard ${ }^{5}$, Tiffany A. Wallace ${ }^{6}$, Jeffrey A. Moscow ${ }^{7}$, James H. Doroshow ${ }^{8}$, Nicholas Mitsiades ${ }^{9}$, Salma Kaochar ${ }^{9}$, Chong-xian Pan ${ }^{4,10}$, Moon S. Chen Jr. ${ }^{4}$, Luis Carvajal-Carmona ${ }^{4}$, Alana L. Welm ${ }^{11}$, Bryan E. Welm ${ }^{11}$, Michael T. Lewis ${ }^{9}$, Ramaswamy Govindan ${ }^{12}$, Li Ding ${ }^{12}$, Shunquang Li ${ }^{12}$, Meenherd Herlyn ${ }^{13}$, Mike Davies ${ }^{14}$, Jack Roth ${ }^{14}$, Funda Meric-

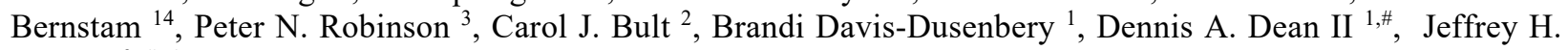
Chuang $3, \#, \&$

1: Seven Bridges, Charlestown, MA.

2: The Jackson Laboratory, Bar Harbor, ME.

3: The Jackson Laboratory for Genomic Medicine, Farmington, CT.

4: University of California - Davis, Davis, CA.

5: Leidos Biomedical Research, Inc, Frederick National Laboratory for Cancer Research, Frederick, MD.

6: Center to Reduce Health Disparities, National Cancer Institute, Bethesda, MD.

7: Investigational Drug Branch, National Cancer Institute, Bethesda, MD.

8: Division of Cancer Treatment and Diagnosis, National Cancer Institute, Bethesda, MD.

9: Baylor College of Medicine, Houston, TX.

10: Harvard Medical School, West Roxbury, MA.

11: Huntsman Cancer Institute, Salt Lake City, UT.

12: Washington University School of Medicine, St. Louis, MO.

13: The Wistar Institute, Philadelphia, PA.

14: The University of Texas MD Anderson Cancer Center, Houston, TX.

* Mr. Koc and Dr. Lloyd are co-first authors providing an equal contribution to the manuscript

\# Drs. Dean and Chuang are co-senior authors providing equivalent oversight to work presented in this manuscript

\& Dr. Chuang is the corresponding author

Keywords:

PDX Models, Tumor Volume Data, RNA-Seq, Whole Exome, and PDMR

\section{Financial Support:}

This work was supported by NIH funding to the PDXNet Data Commons and Coordination Center (NCI U24-CA224067) to the PDX Development and Trial Centers (NCI U54-CA224083, NCI U54-CA224070, NCI U54-CA224065, NCI U54-CA224076, NCI U54-CA233223, and NCI U54-CA233306). The Cancer Genomics Cloud powered by Seven Bridges is a Cancer Research Data Commons Cloud Resource, funded in whole or in part with Federal funds from the National Cancer Institute, National Institutes of Health, Contract No. HHSN261201400008C and ID/IQ Agreement No. $17 X 146$ under Contract No. HHSN261201500003I and 75N91019D00024.

\section{Conflict of Interest:}

The University of Utah may choose to license PDX models developed in the Welm labs, which may result in tangible property royalties to Drs. Welm and members of their lab who developed the models. MTL is a founder and limited partner in StemMed Ltd. and a manager in StemMed Holdings, it's general partner. He is a founder and equity stake holder in Tvardi Theraeutics Inc. Some PDX are exclusively licensed to StemMed Ltd. resulting in royalty income to MTL. Lacey Dobrolecki is a compensated employee of StemMed Ltd. The other authors declare no competing interest.

Word Count: 4,148

Contact information:

Prof. Jeffrey H. Chuang

The Jackson Laboratory for Genomic Medicine

10 Discovery Drive Farmington, CT 06032

Phone: 837-856-2473

Email: jeff.chuang@jax.org
\# of Figures: 4

\# of Tables: 3 
PDXNet Portal

\section{Abstract}

2 We created the PDX Network (PDXNet) Portal (https://portal.pdxnetwork.org/) to centralize 3 access to the National Cancer Institute-funded PDXNet consortium resources (i.e., PDX models, 4 sequencing data, treatment response data, and bioinformatics workflows), to facilitate 5 collaboration among researchers, and to make resources easily available for research. The portal 6 includes sections for resources, analysis results, metrics for PDXNet activities, data processing 7 protocols, and training materials for processing PDX data.

8 The initial portal release highlights PDXNet model and data resources, including 334 new models 9 across 33 cancer types. Tissue samples of these models were deposited in the NCI's Patient10 Derived Model Repository (PDMR) for public access. These models have 2,822 associated 11 sequencing files from 873 samples across 307 patients, which are hosted on the Cancer Genomics 12 Cloud powered by Seven Bridges and the NCI Cancer Data Service for long-term storage and 13 access with dbGaP permissions. The portal also includes results from standardized analysis 14 workflows on PDXNet sequencing files and PDMR data (2,594 samples from 463 patients across 1578 disease types). These 15 analysis workflows for whole-exome and RNA-Seq data are freely 16 available, robust, validated, and standardized.

17 The model and data lists will grow substantially over the next two years and will be continuously 18 updated as new data are available. PDXNet models support multi-agent treatment studies, 19 determination of sensitivity and resistance mechanisms, and preclinical trials. The PDXNet portal 20 is a centralized location for these data and resources, which we expect to be of significant utility 21 for the cancer research community. 
PDXNet Portal

\section{Introduction}

23 Patient-Derived Xenograft (PDX) models are cancer models that support personalized medicine research and preclinical and co-clinical trials ${ }^{1-5}$. Specific PDX research areas include the study of sensitivity and resistance mechanisms, evaluation of new treatment options, and the study of tumor heterogeneity. The PDX research community is rapidly growing, with PDX-generated data being the preferred support for proposing human clinical trials ${ }^{6}$. In 2017, the National Cancer Institute (NCI) funded the PDX Development and Trial Centers (PDTC) research network (PDXNet, pdxnetwork.org) consortium to accelerate PDX research by developing new PDX models across cancer types, identifying new multi-agent treatments to bring forward into clinical trials, generating complementary RNA-Seq and whole-exome sequencing data, and increasing the ethnic diversity of publicly available PDX models.

PDXNet was also charged with developing collaborative research projects involving the 6 different PDTCs to advance PDX science. Each of the PDTCs came into PDXNet with its own home-grown data standards, data analytic pipelines and workflows. To facilitate collaboration, the disparate processes and databases required harmonization at many different steps, so that data from centers could be combined and analyzed efficiently. The harmonization goal was achieved through the creation of the PDXNet portal and the analytical tools within it. The PDXNet portal resources created by this effort enabled the successful completion of several collaborative research projects ${ }^{7-10}$ and are supporting many others.

In addition to facilitating PDXNet research, an additional benefit of the PDXNet Portal is to make the PDXNet-generated data and workflows of the PDXNet Portal available as a public resource. These data will support cancer research by increasing the quantity and diversity of PDX data available and decreasing the effort required to analyze PDX sequencing data. We present the PDXNet Portal as a utility for PDXNet data for the larger scientific community.

There are several existing public PDX resources that complement the PDXNet Portal. Launched in 2012, the NCI Patient-Derived Model Patient Repository (PDMR) ${ }^{11}$ collects and develops PDX models and associated standardized sequencing data (RNA-Seq and whole-exome), with the goal of supporting academic and industry research. The PDMR maintains a publicly available database of models and an File Transfer Protocol (FTP) site for accessing sequencing data. Another resource is PDXFinder ${ }^{12}$. PDXFinder is an online resource that aims to harmonize internationally generated PDX models and their associated metadata. PDXFinder is a collaboration between the European Molecular Biology Laboratory's European Bioinformatics Institute (EMBL-EBI) and the Jackson Laboratory. A key component of data harmonization in PDXFinder is the PDX minimal information standard (PDX-MI) ${ }^{13}$, which allows for standardized PDX information exchange. PDXFinder employs PDX-MI to support complex model searches that enable users to identify model descriptions and subsequently link to model information and a request form. EuroPDX is a consortium of eighteen non-profit cancer institutes that collaborate and coordinate PDX model development and access to improve cancer patients' standard of care. Next, the EuroPDX Data

60 Portal (https://dataportal.europdx.eu/) is a resource that provides information about PDX models 61 generated by EuroPDX researchers and clinicians. Lastly, the Baylor College of Medicine PDX 62 Portal (https://pdxportal.research.bcm.edu/) provides access to breast cancer, leukemia, pediatric liver cancer, pancreatic cancer, and sarcoma model collections. 
The primary aim of the PDXNet Portal is to support the Cancer Moonshot ${ }^{14}$ model and data sharing goals ${ }^{15}$. The PDXNet Portal facilitates the distribution of resources, complementary data analyses, and developed tools. The resources generated include data collections and standardized bioinformatics workflows. Complementary data analyses such as data quality control analyses that support data-use are also available from the portal. Tools developed to support the use of the data (e.g., workflow cost estimation) are also accessible. Integration with the NCI Cloud Resource, the Cancer Genomics Cloud powered by Seven Bridges (CGC) ${ }^{16}$, allows approved researchers to directly analyze PDXNet data or use developed workflows on private data. This manuscript details the PDXNet portal features that provide a gateway for identifying and accessing resources generated by the PDXNet community.

\section{Portal Design and Organization}

The PDXNet Portal is designed to support coordination with other resources, including the PDMR, the CGC, and the NCI Cancer Data Service (CDS) ${ }^{17}$. Currently, the PDXNet portal references PDMR model information, genomic, transcriptomic, and tumor volume response data used in PDXNet research activities. The CGC serves as a PDXNet data staging area supporting data harmonization, standardized data processing, and research activities. The PDXNet Portal supports submission of sequencing data to the CDS to provide long-term research access to PDXNet data resources. The CDS is part of the NCI Cancer Research Data Commons which aims to store data resources generated by NCI-funded research. The CDS is available from across the NCI data infrastructure through a dbGaP access mechanism. The PDXNet portal augments the dbGaP submission process, through an administrative feature for generating data reports and through a $\mathrm{dbGaP}$ submission tool written to support CDS submissions. The PDXNet Portal aims to use data standards when they exist, supporting both the PDX-MI standard ${ }^{13}$ and the PDMR data structures ${ }^{11}$. These existing data structures allow for collaboration and information sharing with existing PDX resources.

\section{Portal Features}

90 The PDXNet Portal is a publicly accessible website (https://portal.pdxnetwork.org/) with the

91 primary function of providing access to the PDXNet models and information on how to obtain sequencing data. We extended the portal's primary mission to include additional resources, including supporting access to the PDMR sequencing file data set, a PDXNet hematoxylin and eosin stain image data set, and PDMR tumor volume data. Below, we describe the features and sections of the Portal in detail.

\section{PDXNet Portal Landing Page}

The PDXNet Portal Landing page includes an overview and summary panel of contents (Figure 1). The Portal overview identifies the primary PDXNet funding sources and participants. In summary, the NCI Cancer Therapy Evaluation Program (CTEP) funds four PDTCs and the PDCCC, whereas the NCI Center to Reduce Cancer Health Disparities funds two PDTCs (see Table 1 for additional details). The portal directs questions and requests for additional information 
The data summary panel on the right side of the screen allows the reader to review model and data summaries and the portal update timeline. The data summary panel lists the number of PDTCs contributing data, the number of files uploaded by the PDTCs and available from the PDMR, the total number of models, and the number of cancer types represented in those models. Tabs allow the reader to review summary figures for PDX Models by cancer type and contributing PDTC, sequencing files by experimental strategy, ancestry, and the Portal Update Timeline.

\section{Resources}

110 The PDXNet Portal resources section includes pages that describe models, data (genomic, 111 transcriptomic, and image), and analysis workflows made available on the CGC by the PDXNet 112 consortium. The CGC based analysis workflows are a significant resource developed by the 113 PDXNet community, allowing for reproducible and standardized analysis of PDX data ${ }^{8}$. The resource section also highlights data mirrored from the PDMR sequencing data repository to the CGC to support research activities. Lastly, we provide interactive plots and tables of sequencing data information for the PDXNet and PDMR sequencing data sets on the CGC. The PDXNet portal presents each resource (e.g., PDXNet, PDMR, workflows) on a separate page.

\section{PDXNet Models}

119 The PDXNet Portal Models tab summarizes verified model submissions to the PDMR made by each PDTC (Supplement Figure 1). The PDXNet models are a primary consortium deliverable. Each model submitted by a PDTC to the PDMR includes a completed model submission form that details the general PDX information, model-specific information, and tissue implantation details. Metadata are consistent with the PDX-MI and the PDMR data format. The metadata includes model id information to facilitate search and cross referencing to related PDMR models.

To date, PDXNet researchers have submitted 334 models to the PDMR across 33 cancer types. $(20.1 \%, 67)$, adenocarcinoma - colon $(12.3 \%, 41)$, and adenocarcinoma - pancreas $(7.8 \%, 26)$. See Table 2 for additional details.

130 The PDXNet Portal summarizes sequencing data submitted by the PDTCs for intraconsortium sharing and for public sharing (Figure 2). The PDXNet Data Collection - PDTC tab presents the core PDXNet sequencing data set. We processed submitted sequencing data with standardized workflows (e.g., whole exome capture; additional) according to a written standard operating

135 for standardized processing.

136 PDXNet researchers contributed 2,822 total sequencing files that include both whole-exome 137 (80.7\%, 2,278) and RNA-Seq $(19.3 \%$, 544) data. Six institutional contributors submitted 873 138 samples from 307 patients. The sequencing sample types include PDX $(51.6 \%, 1457)$, tumor $(28.6$, 139 808), normal $(17 \%, 480)$, and blood $(2.7 \%, 76)$. The most prevalent diseases represented among 140 the samples include breast $(42.1 \%, 1,189)$, lung $(12.8 \%, 362)$, pancreas $(6.2 \%, 174)$, colon $(5.8 \%$, 141 164). Metadata provided by centers did not include disease information for $18.2 \%$ (515) samples 142 (see Table 3 for additional information). 
PDXNet Portal

\section{PDMR Sequencing Data}

The PDXNet Portal Model tab summarizes sequencing data transferred from the PDMR FTP server to the CGC as of August 2020 (Supplement Figure 2). The PDMR generates whole-exome and transcriptome sequencing data from models submitted according to tissue collection best practices and model quality control practices ${ }^{11}$. Molecular characterizations include whole-exome sequencing and gene expression profiling. We processed the PDMR sequencing data with standardized workflows as for the PDTC data.

The PDMR sequencing dataset on the CGC includes 9,492 paired-end sequencing files that include both whole-exome $(52.8 \%, 5,012)$ and RNA-Seq $(47.2 \%, 4,480)$ data (See Supplement Table 4). The data set includes 2,594 samples from 445 patients covering 34 disease types. The sequencing sample types include PDX $(82.7 \%, 7,846)$, primary tumor $(5.7 \%, 542)$, normal germline $(5.5 \%$, $520)$, and organoid $(3.2 \%, 304)$. The most prevalent diseases represented among the samples include colon $(21.1 \%, 2,002)$, head and neck $(11.6 \%, 1,102)$, soft tissue neoplasm $(10.1 \%, 958)$, skin $(8.7 \%, 828)$. Due to the size and cost associated with data transfers, synchronization between the PDMR sequencing database and the CGC dataset is done periodically. The PDMR data webpage has the most updated list of available PDMR sequencing data processed with standardized PDXNet workflows.

\section{PDMR Image data}

The PDXNet Portal Image tab summarizes hematoxylin-eosin stain (H\&E) image data provided by the PDMR (Supplement Figure 3). The PDMR image data on the CGC includes 593 images scanned from PDX $(93.8 \%, 556)$ and primary tumors $(6.2 \%, 37)$. The images correspond to 593 samples taken from 92 patients across 37 disease types. The PDX passages ranged from P0 to P6 with the top four passages corresponding to P1(41.4\%, 225), P2 (24.1\%, 131), P0 (22.8\%, 124), and P3 $(8.3 \%, 45)$. The PDXNet Portal currently supports 43 metadata fields that data submitters can populate upon submission (See Supplement Table 1 for the complete list).

\section{Interactive Data Explorers}

The PDXNet Portal data explorer allows users to interactively create summary tables from the PDXNet and PDMR sequencing datasets (Supplement Figure 4). The interactive table supports 10 table and chart types including simple tables, bar charts, line charts, and heat maps (see Supplement Table 1 for full list). Interactive tables also support 22 data summary options including count, sum, average, and variance (see Supplement Table 2 for the full list). The user drags and drops from 20 metadata fields to the table type area to construct the table. Metadata field examples include contributor, sample type, experimental strategy, and passage (see Supplement Table 3 for complete list).

\section{PDXNet Workflows}

The PDXNet Portal Workflows tab summarizes analysis workflows developed by the PDXNet community (Supplement Figure 5). We selected workflows for standard consortium-wide data processing and public release from those submitted by each PDTC after benchmarking with simulated and experimentally derived PDX data ${ }^{8}$. Since the initial public release, we have restructured the workflows to efficiently process normal (tissue), tumor-only, and tumor-normal data. These workflows are implemented on the CGC using the Common Workflow Language $(\mathrm{CWL})^{18}$ with Docker containerized tools, which allows for easy sharing and analysis reproducibility. The PDXNet consortium developed a set of 15 workflows validated for processing 
of both whole-exome and RNA-Seq data (Supplement Table 4). We are sharing these validated and tested workflows with the broader community via the CGC Public Apps Gallery (https://cgc.sbgenomics.com/public/apps\#q?search=pdx). The use of CWL allows these workflows to be portable to any CWL-compliant execution environment. The workflows collectively facilitate the analysis of whole-exome or RNA-Seq data via mouse read disambiguation, read alignment, variant calling or transcript quantification, and sample and cohort level quality control. For whole-exome data, we also compute copy number variation (CNV), tumor mutational burden (TMB), microsatellite instability (MSI), and homologous recombination deficiency (HRD) during standardized processing. A full explanation of inputs, outputs, and data processing steps for each workflow is provided on the CGC in the respective description section.

\section{Analysis}

The PDXNet Portal analysis section includes several metrics derived from primary data sources and are described below in more detail. These results were generated from standard processing analysis workflows or through PDXNet research activities ${ }^{8}$, and we provide these analyses to support independent research by the broader research community.

\section{Ancestry Analysis}

The PDXNet Portal Ancestry Analysis page summarizes genetic ancestry analysis for datasets on the portal (Supplement Figure 6). We compute ancestry with SNPweight ${ }^{19}$ using a reference dataset generated from the 1000 Genomes Project Phase $\mathrm{III}^{20}$. We classify each sample into one of five categories, which correspond roughly to the concept of "continental ancestry." ${ }^{21}$ These categories include European (EUR), African (AFR), American (AMR), East Asia (EAS), and South Asian (SAS). Samples that could not be confidently assigned to one of these categories are labeled Mixed (MIX). On the left side of the page, ancestry data filters allow the user to select the data contributors, ancestry, and disease type. Applying the selected filter to the data regenerates the two summary figures. The first summary figure is a bar chart that shows the ancestry distribution for the selected disease types. The second summary figure shows a pie chart with each slice corresponding to the ancestry types chosen. Supplement Table 6 shows the summary of ancestry estimation from PDX Models submitted to the PDMR.

\section{HRD-TMB-MSI Analysis}

The PDXNet Portal HRD-HSI-TMB analysis page allows the user to filter and summarize three computational metrics generated from whole-exome sequencing data by PDXNet standardized processing (Figure 3). The three computed metrics are Homologous Recombination Deficiency (HRD), Tumor Mutational Burden (TMB), and Microsatellite Instability (MSI). HRD is computed with ScarHRD ${ }^{22}$ for matched normal data. TMB is calculated as the number of coding mutations that meet all quality criteria per $\mathrm{Mb}$ of the genome. Quality criteria are assessed using coverage, allele frequency, mapping quality, and strand bias. Variants included in the calculation are somatic and non-polymorphic, and are defined in SnpEff' ${ }^{23}$ as 'high' or 'moderate' functional impact. As only a portion of the genome was sequenced, genome coverage $(\mathrm{Mb})$ is calculated from the input target coverage BED file. MSI is calculated with MANTIS ${ }^{24}$ for samples with matched normal data, and calculated with MSIsensor $2^{25}$ for tumor-only samples. For each metric, users can set data filters for the visualizations. The data filters, on the left side of the page, allow the user to select data contributor, sample type, experimental strategy, and disease type. Applying the selected filter to the data generates a boxplot chart displaying the selected metrics for each disease type chosen (Figure 3). 
PDXNet Portal

\section{Tumor Volume Analysis}

The PDXNet Portal Tumor Volume Analysis Page allows the user to visualize raw tumor volume growth data provided by the PDMR (Figure 4). The filters enable the user to select contributor, treatment, and disease type on the page's left side. Applying the selected filter to the data regenerates the Tumor Volume and the Tumor Disease Types figure tabs. The currently available volume data is from 75 models representing 30 disease types, which were treated with seven possible agents (Supplement Table 7). The dataset has 17,920 volume measures from 89 treatment studies. The Tumor Volume tab allows the user to choose plot level (Animal and Treatment Arm) and plot pattern (multiple and combined), reorganizing the plots to correspond to select values. The Tumor Disease type tab plots a disease pie chart based on user selection (Figure 4).

\section{Quality Control Analysis}

The PDXNet Portal QC Analysis page provides plots and tabular results for selected QC metrics (Supplement Figure 7). The page displays QC metrics generated during the standardized data processing procedure for each relevant data type. The page provides sub-tabs showing wholeexome and RNA-Seq quality control metrics for a selected dataset PDXNet or PDMR. The wholeexome tabs plot mean target coverage, percent target bases with greater than $20 \%$ coverage, and percent duplication by data contributor. The RNA-Seq tabs plot percent usable bases, percent ribosomal bases, and percent correct strand reads. The plotted metrics, along with additional QC metrics are available in a table at the bottom of the page.

\section{Tools}

The PDXNet Portal tools section includes several Portal specific tools developed to support present and future PDXNet and other independent general research activities.

\section{Workflow Cost prediction}

The Workflow cost prediction tool allows users to estimate the cost of processing their samples on the CGC with the PDXNet workflows. This tool uses prediction models (gradient boosting trees ${ }^{26}$ ) generated from 7,000 workflow runs. The user can select either whole-exome or RNA-Seq workflows and provide the number and optionally size of files to process. The calculator computes the storage and computation cost for processing the user defined dataset. The estimated costs assume the workflows were run on the CGC using spot instances. We expect the tool to allow users to estimate data storage and computational cost for their own analyses allowing for estimating grant budgets and budgeting lab expenses.

\section{PDX Minimum Information Metadata - Creation}

The generate metadata tab allows users to interactively generate a PDX minimum information metadata sheet (PDX-MI). As described above, the PDXFinder working group developed the PDX-MI as a standard for exchanging PDX information among institutions. The generate metadata tab allows the user to create a PDX-MI spreadsheet by stepping through data entry dialog boxes. The interface supports entry of patient information, treatment information, tumor information, model, and sequencing metadata. The user downloads the spreadsheet upon data entry completion, and no information is stored permanently on the PDXNet Portal site.

\section{PDX Minimum Information Metadata - Validation}

The validate metadata tab allows users to upload and validate a PDX-MI metadata spreadsheet. The user can review uploaded contents at the bottom of the page. The validation verifies that 
PDXNet Portal

required metadata fields are present and that entries are valid. The validation feature generates a summary of required fields that includes percent completed and most common data entry per field. The validation feature reduces the amount of time necessary to review and check submitted PDXMI spreadsheets.

\section{Implementation}

The backend of the PDXNet Portal is an R-Shiny app hosted on a cloud-based server. The portal uses the PDMR and PDX-MI metadata standards. The PDMR and PDX-MI standards allow us to harmonize data across sources, quickly import data from the PDMR and other data sources, and exchange information with other PDX related portals. We also collect additional metadata required to facilitate computation on the CGC, including omics-related information. We use the Cancer Therapy Evaluation Program (CTEP) disease classification to standardize disease entries although we initially accepted institutionally-defined disease classification. In the cases where a standard does not exist; we collect sufficient metadata required to display and process the data source. For example, we take a minimalistic approach to managing tumor volume and H\&E image data. Several PDXNet teams are working towards the development of best practices for these data types. Until these best practices are published, we will evolve these operational standards to support harmonization and analysis.

The PDXNet Portal team updates information on the portal semi-automatically using the same data model as the PDMR, allowing PDXNet to sync with PDMR model information. The PDMR provides regular updates to PDXNet on PDTC model submissions to update the PDX Model's page. We receive sequencing data upload updates from the PDTCs and the PDMR, and we have developed scripts for extracting PDXNet standardized processing results, allowing for quality control information and computational metrics to be tabulated for semi-automated PDXNet Portal updates. The PDXNet Portal source code will not be made publicly available for security reasons. Future PDXNet Portal versions will support controlled access sign-in to provide links to controlled files.

\section{Data Availability}

Each PDXNet Portal data tab allows users to download metadata. Data for smaller data types such as tumor volume data and computed metrics (ex. HRD and TMB) can be downloaded directly from the portal. For larger data types, please request data from the PDXNet Portal's Contact page. We will coordinate with PDTCs to make data available either directly or through dbGaP as required by the PDXNet data sharing agreement.

\section{Discussion}

The PDXNet Portal is a vital component of the PDXNet consortium. The portal establishes a mechanism for public discovery of consortium-generated resources including models, data, and workflows. The portal allows researchers to examine the data, models, and metadata using integrated query features. These portal capabilities facilitate cancer data discovery, a core goal of the NCI Cancer Moonshot program. Additionally, the PDXNet Portal allows the consortium to manage data analysis projects by clarifying which data are available, their source, their quality, and their suitability for research projects and scientific questions. Within the PDXNet consortium, the PDXNet Portal functions as a centralized source of information for the status of the available 
PDXNet Portal

models. The standardized sequence processing, quality control, and computation of common metrics (e.g., MSI, TMB, HRD, and genetic ancestry) further enhance data analysis, model use, prioritization of future models and data collection.

Enhancement of collaboration between researchers is a main objective of the PDXNet Portal. To accomplish this, we are integrating the PDXNet Portal and PDXNet data with existing NCI, NIH, and NCBI infrastructure. All data visible on the PDXNet Portal will also be available through the Cancer Data Service (CDS) ${ }^{17}$ through $\mathrm{dbGaP}^{27}$ access. Accessing PDXNet data on the Cancer Genomics Cloud ${ }^{28}$ allows users to perform sophisticated analyses utilizing cloud computing within an integrated bioinformatics ecosystem. By co-locating data and analysis, as well as integrating data management, this infrastructure can decrease the time required for researchers to perform analyses.

For large consortia such as PDXNet, metadata and secondary data types are often just as important as sequencing data for supporting impactful research. Examples of these additional data types include high-resolution images and tumor volume/drug response data. These data extend the types of problems researchers can address. We expect that the portal's image and tumor volume functionality will expand as these datasets grow. Future iterations of the portal will include interactive exploration across data types allowing users to address complex research problems.

PDX models are widely used in cancer research, but there remain challenges in standards for data submission, access, and quality. The PDXNet Portal reflects PDXNet activities to implement such standards not only for sequencing data but also metadata and secondary data types. Another consideration requiring careful implementation is to balance data security versus ease of use. The PDXNet Portal will grow with new data and features as the PDXNet consortium continues to generate new models. Consequently, standardized processing and batch effects are of increasing concern for downstream analyses. To ensure that researchers have confidence in the data quality, we will continue to share the informative metrics computed by the standardized PDXNet quality control workflows.

Several key features are the focus for the next iteration of the PDXNet Portal. These include tools to search for commonly found genomic variants (ex. SNPs, INDELs, and copy number variations) within models, diseases and genes of interest, and interactive exploration of gene expression data. These tools would enable researchers to perform meaningful analyses directly from the portal and more rapidly realize value from PDXNet data. Visualization and analysis of associated data, including imaging and tumor volume/drug response data, will also be a focus. These data types have the potential for high impact, particularly given the innovation in large scale data visualization techniques in many fields.

Further development of links between the PDXNet Portal and NCI computational infrastructure will benefit researchers as well. Moving large quantities of data is time-consuming and can be expensive. Enabling researchers to perform their analysis where the data is already present lowers the entry barrier into the computational analysis of PDX model data. To facilitate these links, we envision users will be able to create cohorts for analysis using the PDXNet Portal and transferring that selection to the Cancer Genomics Cloud or other computational platform, where they will be able to easily take advantage of well-developed computational infrastructure. 
PDXNet Portal

354 We will extend PDXNet Portal capabilities as the size and complexity of PDXNet datasets grow. 355 These enhancements will allow the research community to quickly find and evaluate PDXNet 356 resources to supplement their research studies. We will continue to improve the PDXNet Portal 357 value by collaborating with related PDX initiatives, including the PDMR, PDXFinder, and 358 EuroPDX. Such collaborations will demonstrate how to effectively conduct studies across 359 institutions, providing examples for the broader research community in how to optimize their PDX 360 studies with respect to the public PDX models and datasets that are becoming increasingly 361 available.

\section{Acknowledgments}

363 We would like to thank the patients who provided the tissues that support the PDXNet model and 364 sequencing data generation.

365 This work was supported by NIH funding to the PDXNet Data Commons and Coordination Center 366 (NCI U24-CA224067) to the PDX Development and Trial Centers (NCI U54-CA224083, NCI 367 U54-CA224070, NCI U54-CA224065, NCI U54-CA224076, NCI U54-CA233223, and NCI U54368 CA233306). The Cancer Genomics Cloud powered by Seven Bridges is a Cancer Research Data 369 Commons Cloud Resource, funded in whole or in part with Federal funds from the National Cancer 370 Institute, National Institutes of Health, Contract No. HHSN261201400008C and ID/IQ Agreement 371 No. 17X146 under Contract No. HHSN261201500003I and 75N91019D00024. 
PDXNet Portal

\section{References}

377 1. Koga, Y. \& Ochiai, A. Systematic Review of Patient-Derived Xenograft Models for

$378 \quad$ Preclinical Studies of Anti-Cancer Drugs in Solid Tumors. Cells 8, 418 (2019).

379 2. Dobrolecki, L., Airhart, S., Alferez, D. \& Aparicio, S. Patient-derived Xenograft (PDX)

$380 \quad$ models in basic and translational breast cancer research. Cancer Metastasis Rev. 35, 547-

381

382

383 573 (2016).

3. Collins, A. T. \& Lang, S. H. A systematic review of the validity of patient derived xenograft (PDX) models: The implications for translational research and personalised medicine. PeerJ 2018, 1-22 (2018).

4. Brown, K. M. et al. Patient-derived xenograft models of colorectal cancer in preclinical research: A systematic review. Oncotarget 7, 66212-66225 (2016).

5. Grandori, C. \& Kemp, C. Personalized cancer moels for target discovery and precision medicine. Trends Cancer 4, 634-642 (2018).

6. Hidalgo, M. et al. Patient-Derived Xenograft Models: An Emerging Platform for Translational Cancer Research. Cancer Discovery 4, 998-1013 (2014).

7. Woo, X. Y. et al. Genomic data analysis workflows for tumors from patient-derived xenografts (PDXs): Challenges and guidelines. BMC Medical Genomics 12, 1-19 (2019).

8. Evrard, Y. A. et al. Systematic establishment of robustness and standards in patientderived xenograft experiments and analysis. Cancer Research 80, 2286-2297 (2020).

9. Sun, H. et al. Comprehensive characterization of 536 patient-derived xenograft models prioritizes candidates for targeted treatment. Nature Communications 12, (2021).

10. Woo, X. Y. et al. Conservation of copy number profiles during engraftment and passaging of patient-derived cancer xenografts. Nature Genetics 53, 86-99 (2021).

11. The NCI Patient-Derived Models Repository (PDMR). Research, NCI-Frederick:

Frederick National Laboratory for Cancer https://pdmr.cancer.gov/ (2021).

12. Conte, N. et al. PDX Finder: A portal for patient-derived tumor xenograft model discovery. Nucleic Acids Research 47, D1073-D1079 (2019).

13. Meehan, T. F. et al. PDX-MI: Minimal information for patient-derived tumor xenograft models. Cancer Research 77, e62-e66 (2017).

14. Lowy, D. \& Collins, F. Aiming High - Changing the Trajectory for Cancer. NEJM 374, 1901-1904 (2016).

15. Sharpless, N. E. \& Singer, D. S. Progress and potential: the Cancer Moonshot. Cancer Cell 1-6 (2021) doi:10.1016/j.ccell.2021.04.015.

16. Lau, J. W. et al. The cancer genomics cloud: Collaborative, reproducible, and democratized - A new paradigm in large-scale computational research. Cancer Research 77, e3-e6 (2017).

17. NCI. Cancer Data Service. https://datacommons.cancer.gov/repository/cancer-data-service (2021).

18. Amstutz, P., Crusoe, M. \& Tijianic, N. Common Workflow lanaguage (CWL) Workflow Description, v1.0.2. (2016).

19. Chen, C. Y. et al. Improved ancestry inference using weights from external reference panels. Bioinformatics 29, 1399-1406 (2013). 
20. Auton, A. et al. A global reference for human genetic variation. Nature 526, 68-74 (2015).

21. Weiss, K. M. \& Long, J. C. Non-Darwinian estimation: My ancestors, my genes'

22. Sztupinszki, Z. et al. Migrating the SNP array-based homologous recombination deficiency measures to next generation sequencing data of breast cancer. npj Breast Cancer 4, 8-11 (2018).

23. Cingolani, P. et al. A program for annotating and predicting the effects of single nucleotide polymorphisms, SnpEff: SNPs in the genome of Drosophila melanogaster strain w1118; iso-2; iso-3. Fly 6, 80-92 (2012).

24. Kautto, E. A. et al. Performance evaluation for rapid detection of pan-cancer microsatellite instability with MANTIS. Oncotarget 8, 7452-7463 (2017).

25. Niu, B. et al. MSIsensor: Microsatellite instability detection using paired tumor-normal sequence data. Bioinformatics 30, 1015-1016 (2014).

26. Hastie, T., Tibshirani, R. \& Friedman, J. Boosting and Additive Trees. in The Elements of Statistical Learning (Springer, 2009).

27. Mailman, M. D. et al. The NCBI dbGaP database of genotypes and phenotypes. Nature Genetics 39, 1181-1186 (2007).

28. Lau, J. W. et al. The cancer genomics cloud: Collaborative, reproducible, and democratized - A new paradigm in large-scale computational research. Cancer Research 77, e3-e6 (2017).

\section{Table 1. PDXNet Development and Trial Centers (PDTC) and the PDX Data Commons} and Coordinating Center (PDCCC)

\begin{tabular}{|c|c|}
\hline & PDXNet \\
\hline \multicolumn{2}{|c|}{ PDX Development and Trials Centers (PDTC) } \\
\hline HCI-BCM* & Huntsman Cancer Institute and Baylor College of Medicine \\
\hline MDACC & MD Anderson Cancer Center \\
\hline WUSTL* & Washington University at St. Louis \\
\hline WISTAR* & Wistar Institute and MD Anderson Cancer Center \\
\hline $\mathrm{BCM}^{\&}$ & Baylor College of Medicine \\
\hline UCDAVIS $^{\&}$ & The University of California at Davis \\
\hline \multicolumn{2}{|c|}{ PDX Data Commons and Coordinating Center } \\
\hline $\mathrm{JAX}-\mathrm{SB}^{* \&}$ & Jackson Laboratory and Seven Bridges \\
\hline
\end{tabular}


PDXNet Portal

\section{Table 2. PDX models generated by PDX Development and Trials Centers}

\begin{tabular}{|c|c|c|c|c|c|c|c|}
\hline & HCI-BCM & MDACC & WUSTL & Wistar & UC Davis & BCM & Totals \\
\hline Breast & 78 & 12 & 2 & 0 & 0 & 16 & 108 \\
\hline Head and Neck & 0 & 0 & 2 & 0 & 0 & 0 & 2 \\
\hline Digestive/Gastrointestinal & 0 & 50 & 44 & 0 & 3 & 0 & 97 \\
\hline Endocrine and Neuroendocrine & 0 & 2 & 0 & 0 & 0 & 0 & 2 \\
\hline Musculoskeletal & 0 & 1 & 4 & 0 & 0 & 0 & 5 \\
\hline Respiratory/Thoracic & 0 & 30 & 1 & 0 & 2 & 0 & 33 \\
\hline Skin & 0 & 3 & 2 & 63 & 0 & 0 & 68 \\
\hline Genitourinary & 0 & 0 & 5 & 0 & 10 & 0 & 15 \\
\hline Gynecologic & 0 & 3 & 0 & 0 & 0 & 0 & 3 \\
\hline Unknown Primary & 0 & 0 & 0 & 1 & 0 & 0 & 1 \\
\hline total & 78 & 101 & 60 & 64 & 15 & 16 & 334 \\
\hline
\end{tabular}

HCI-BCM: Huntsman Cancer Institute and Baylor College of Medicine, MDACC: MD Anderson Cancer Center, WUSTL: Washington University at St. Louis, Wistar: The Wistar Institute, UC Davis: University of California Davis, BCM: Baylor College of Medicine 
bioRxiv preprint doi: https://doi org/10.1101/2021.10.15.464537: this version posted October 28,2021 . The copyright holder for this preprint (which was not certified by peer review) is the author/funder, who has granted bioRxiv a license to display the preprint in perpetuity. It is made available under aCC-BY-NC-ND 4.0 International license.

PDXNet Portal

447 Table 3. Sequencing data files generated by PDX Development and Trial Centers

\begin{tabular}{|c|c|c|c|c|c|c|}
\hline & $\begin{array}{l}\text { Overall } \\
N=2822\end{array}$ & $\begin{array}{c}\text { ВСМ-HCI } \\
\mathrm{N}=1099\end{array}$ & $\begin{array}{c}\text { MDACC } \\
\mathrm{N}=418\end{array}$ & $\begin{array}{c}\text { UC Davis } \\
\qquad \mathrm{N}=48\end{array}$ & $\begin{array}{c}\text { WISTAR } \\
\mathrm{N}=382\end{array}$ & $\begin{array}{c}\text { WUSTL } \\
\mathrm{N}=875\end{array}$ \\
\hline \multicolumn{7}{|c|}{ Experimental Strategy n(\%) } \\
\hline RNA-Seq & $544(19 \%)$ & $102(9 \%)$ & $6(1 \%)$ & $32(67 \%)$ & $144(38 \%)$ & $260(30 \%)$ \\
\hline WES & $2,278(81 \%)$ & $997(91 \%)$ & $412(99 \%)$ & $16(33 \%)$ & $238(62 \%)$ & $615(70 \%)$ \\
\hline \multicolumn{7}{|l|}{ Disease Type, n(\%) } \\
\hline Bladder & $100(3.5 \%)$ & $2(<0.2 \%)$ & $0(0 \%)$ & $48(100 \%)$ & $0(0 \%)$ & $50(5.7 \%)$ \\
\hline Blood & $84(3.0 \%)$ & $0(0 \%)$ & $84(20 \%)$ & $0(0 \%)$ & $0(0 \%)$ & $0(0 \%)$ \\
\hline Bone & $8(0.3 \%)$ & $0(0 \%)$ & $0(0 \%)$ & $0(0 \%)$ & $0(0 \%)$ & $8(0.9 \%)$ \\
\hline Breast & $1189(42 \%)$ & $985(90 \%)$ & $0(0 \%)$ & $0(0 \%)$ & $0(0 \%)$ & $204(23 \%)$ \\
\hline Colon & $164(5.8 \%)$ & $4(0.4 \%)$ & $0(0 \%)$ & $0(0 \%)$ & $0(0 \%)$ & $160(18 \%)$ \\
\hline Gastrointestinal & $22(0.8 \%)$ & $0(0 \%)$ & $0(0 \%)$ & $0(0 \%)$ & $0(0 \%)$ & $22(2.5 \%)$ \\
\hline Head and Neck & $26(0.9 \%)$ & $2(<0.2 \%)$ & $0(0 \%)$ & $0(0 \%)$ & $0(0 \%)$ & $24(2.7 \%)$ \\
\hline Kidney & $58(2.1 \%)$ & $0(0 \%)$ & $0(0 \%)$ & $0(0 \%)$ & $0(0 \%)$ & $58(6.6 \%)$ \\
\hline Lung & $362(13 \%)$ & $0(0 \%)$ & $320(77 \%)$ & $0(0 \%)$ & $0(0 \%)$ & $42(4.8 \%)$ \\
\hline Neuroendocrinal & $2(<0.1 \%)$ & $0(0 \%)$ & $2(0.5 \%)$ & $0(0 \%)$ & $0(0 \%)$ & $0(0 \%)$ \\
\hline Ovarian & $8(0.3 \%)$ & $0(0 \%)$ & $0(0 \%)$ & $0(0 \%)$ & $0(0 \%)$ & $8(0.9 \%)$ \\
\hline Pancreas & $174(6.2 \%)$ & $0(0 \%)$ & $0(0 \%)$ & $0(0 \%)$ & $0(0 \%)$ & $174(20 \%)$ \\
\hline Rectum & $22(0.8 \%)$ & $0(0 \%)$ & $0(0 \%)$ & $0(0 \%)$ & $0(0 \%)$ & $22(2.5 \%)$ \\
\hline Skin & $32(1.1 \%)$ & $0(0 \%)$ & $0(0 \%)$ & $0(0 \%)$ & $0(0 \%)$ & $32(3.7 \%)$ \\
\hline Soft Tissue Neoplasm & $56(2.0 \%)$ & $0(0 \%)$ & $0(0 \%)$ & $0(0 \%)$ & $0(0 \%)$ & $56(6.4 \%)$ \\
\hline Unknown & $515(18 \%)$ & $106(10 \%)$ & $12(2.9 \%)$ & $0(0 \%)$ & $382(100 \%)$ & $15(1.7 \%)$ \\
\hline \multicolumn{7}{|l|}{ Sample Type, n(\%) } \\
\hline Normal & $481(17 \%)$ & $121(11 \%)$ & $142(34 \%)$ & $0(0 \%)$ & $0(0 \%)$ & $218(25 \%)$ \\
\hline PDX & $1,497(52 \%)$ & $594(54 \%)$ & $146(35 \%)$ & $0(0 \%)$ & $202(53 \%)$ & $515(59 \%)$ \\
\hline Tumor & $884(31 \%)$ & $384(35 \%)$ & $130(31 \%)$ & $48(100)$ & $180(47 \%)$ & $142(16 \%)$ \\
\hline
\end{tabular}

HCI-BCM: Huntsman Cancer Institute and Baylor College of Medicine, MDACC: MD Anderson Cancer Center, WUSTL: Washington University at St. Louis, Wistar: The Wistar Institute, UC Davis: University of California Davis, BCM: Baylor College of Medicine 
bioRxiv preprint doi: https://doi.org/10.1101/2021.10.15.464537: this version posted October 28,2021 . The copyright holder for this preprint (which was not certified by peer review) is the author/funder, who has granted bioRxiv a license to display the preprint in perpetuity. It is made available under aCC-BY-NC-ND 4.0 International license.

PDXNet Portal

\section{$450 \quad$ Figures}

451
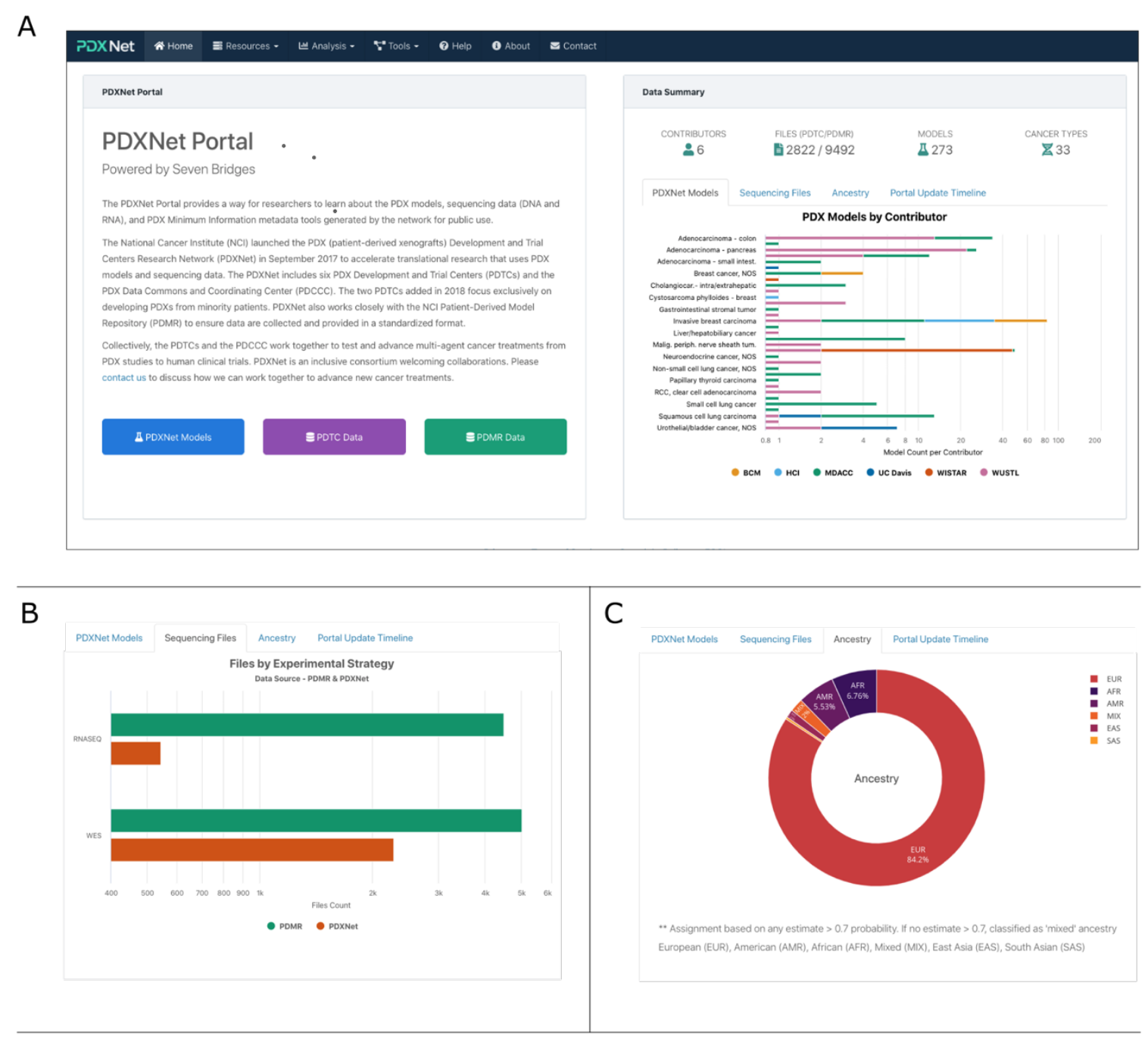

D

\begin{tabular}{ll|l} 
PDXNet Models Sequencing Files Ancestry Portal Update Timeline &
\end{tabular}

Timeline of PDXNet Portal Updates

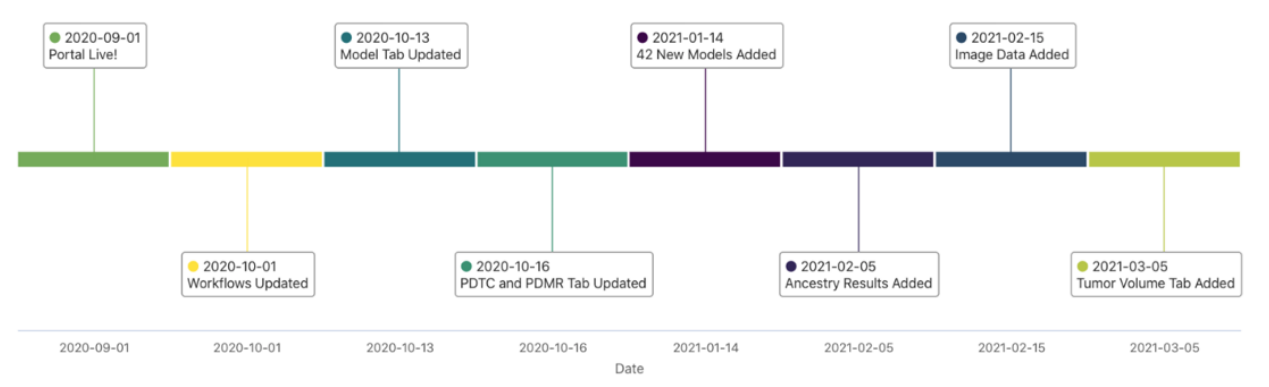

452

453 Figure 1. PDXNet Portal Landing Page 
bioRxiv preprint doi: https://doi.org/10.1101/2021.10.15.464537; this version posted October 28, 2021. The copyright holder for this preprint (which was not certified by peer review) is the author/funder, who has granted bioRxiv a license to display the preprint in perpetuity. It is made available under aCC-BY-NC-ND 4.0 International license.

PDXNet Portal

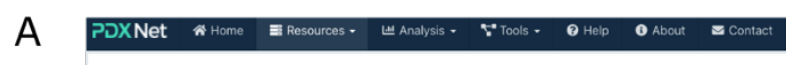

POXNet Sequencing Data Summary - POTC

The PDXNet Portal Data tab summarizes DNA and RNA sequencing fles uploaded to the Cancer Genomics Cloud by the PDX Data and Trial Centers. The page includes data uploads through August 2019. Additional instructions will be provided for accessing the sequncing data on the Cancer Genomics Cloud once we complete the submission to db GaP. Note that semi-annual updates to this page are delayed due to COVID-19 related project priority shifts

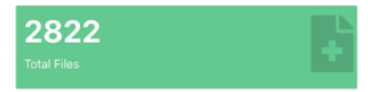

Approximate values, empty metafield cells are not counted!
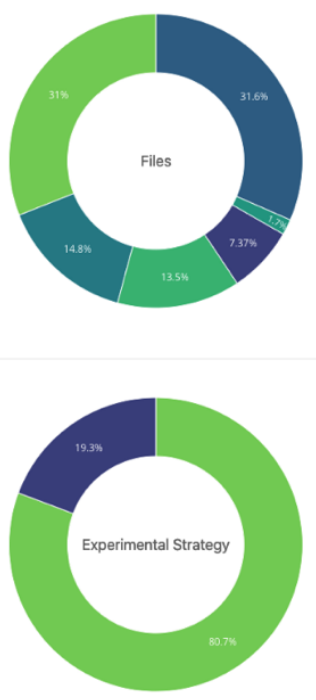

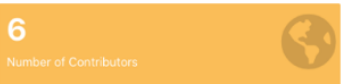

\section{3}

Total Samples
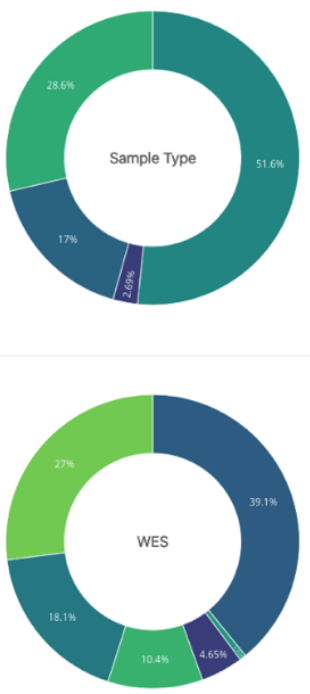

\section{7}

Total patants
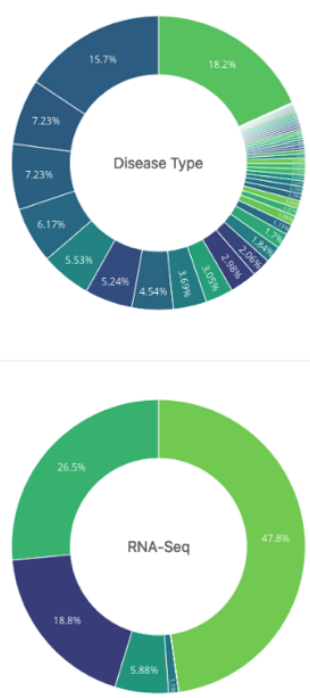

B 
bioRxiv preprint doi: https://doi.org/10.1101/2021.10.15.464537; this version posted October 28, 2021. The copyright holder for this preprint (which was not certified by peer review) is the author/funder, who has granted bioRxiv a license to display the preprint in perpetuity. It is made available under aCC-BY-NC-ND 4.0 International license.

PDXNet Portal

A

HRD - Disease Type

HRD - Disease Type

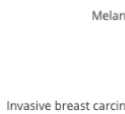

Adenocarcinoma - rectum

Adenocarcinoma - pancreas

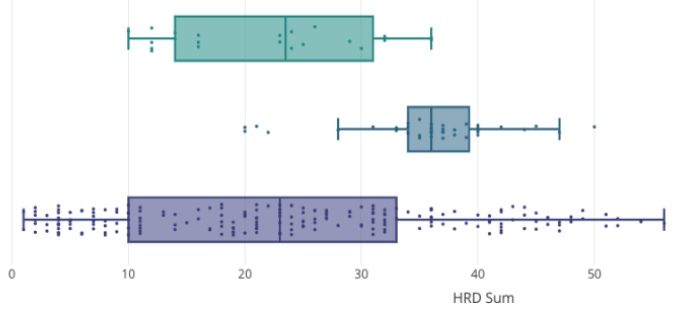

B

TMB - Disease Type

TMB - Disease Type
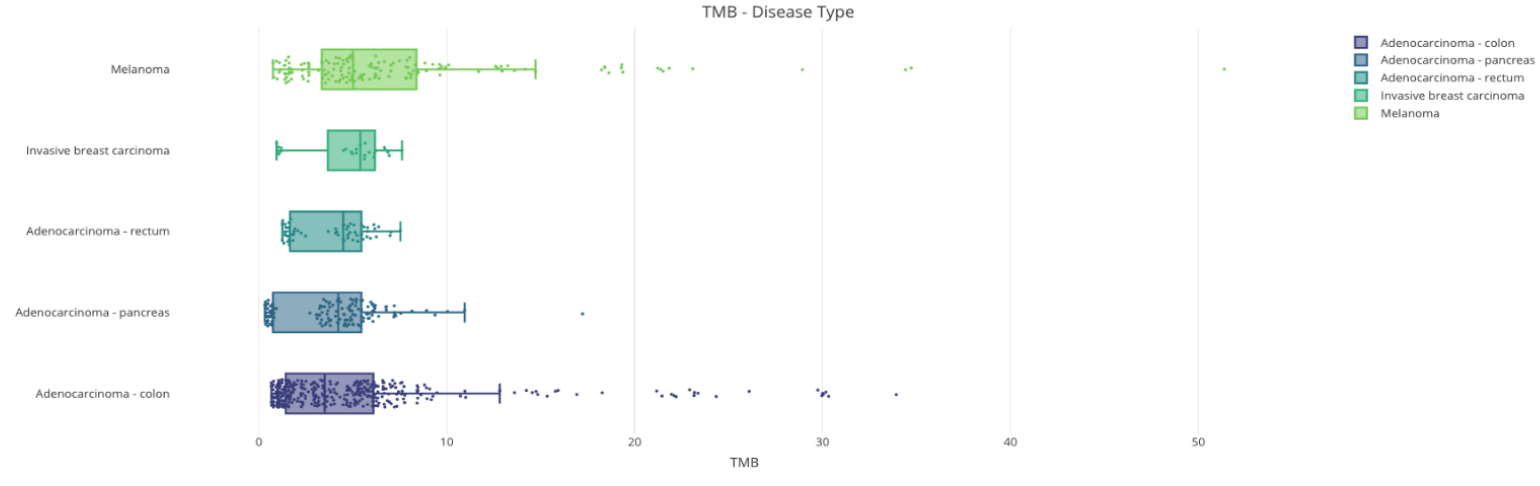

C

MSI (Tumor-only) - Disease Type

MSI (Tumor-only) - Disease Type

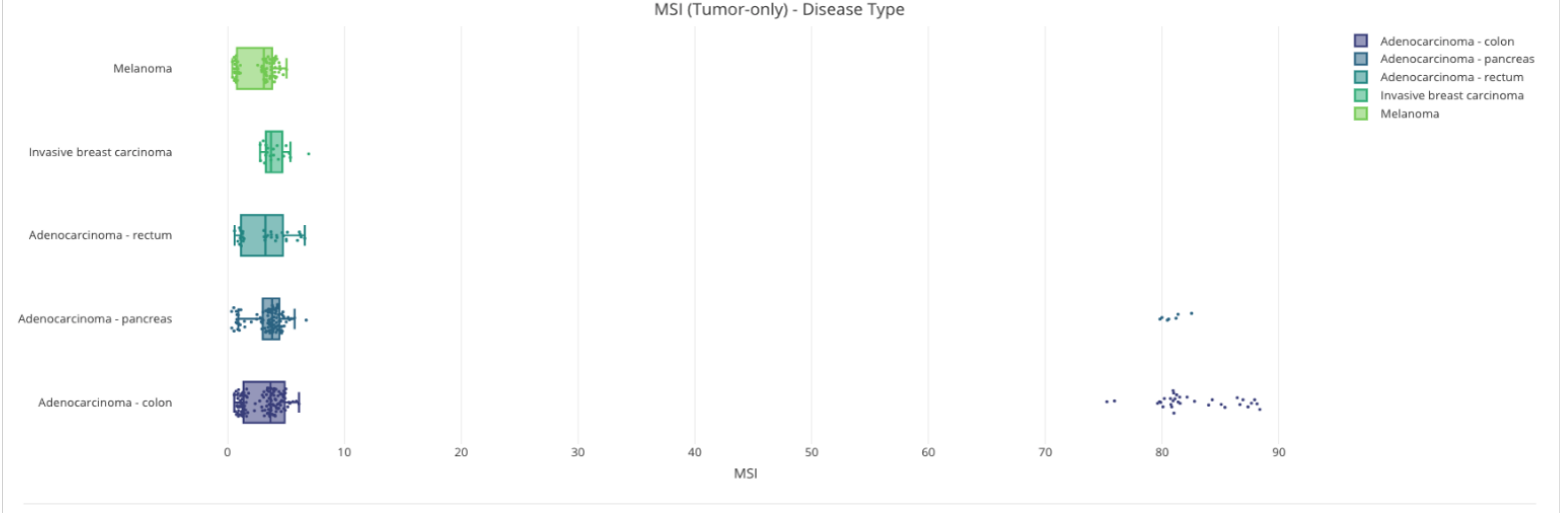


bioRxiv preprint doi: https://doi.org/10.1101/2021.10.15.464537 this version posted October 28,2021 . The copyright holder for this preprint (which was not certified by peer review) is the author/funder, who has granted bioRxiv a license to display the preprint in perpetuity. It is made available under aCC-BY-NC-ND 4.0 International license.

PDXNet Portal

A

Tumor Volume - Summary
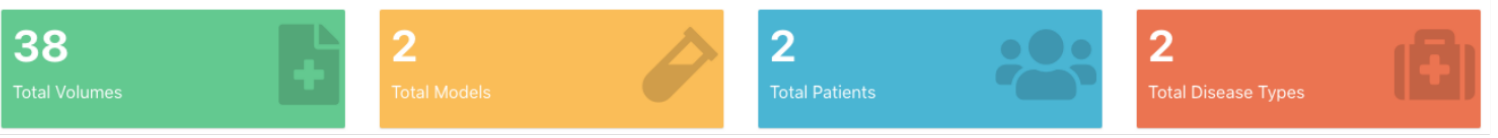

B
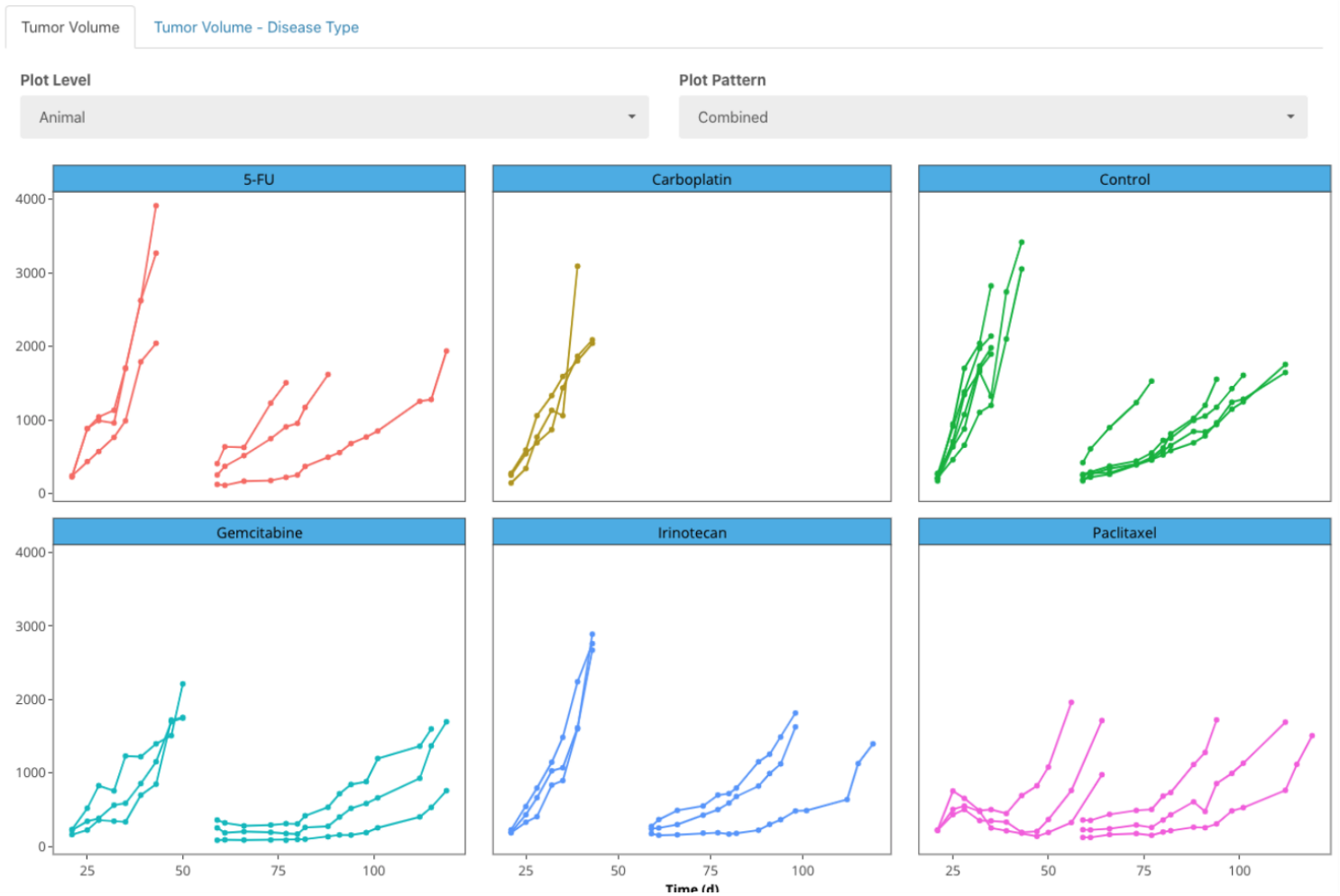

C

Tumor Volume - Data Table

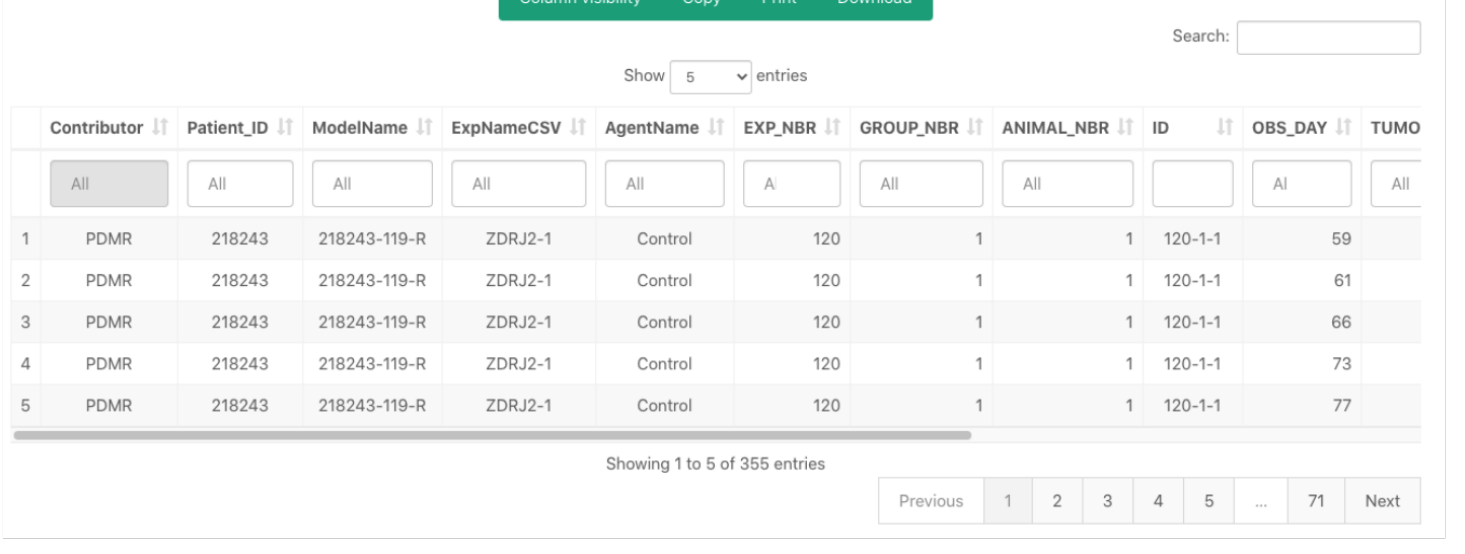


PDXNet Portal

\section{Figure Legend}

\section{Figure 1. PDXNet Portal Landing Page}

465 Views from the PDXNet Portal Landing Page. (A) The initial PDXNet Portal Landing Page. (B)

466 Experimental strategies (whole-exome and RNA-Seq) for the PDXNet (green) and PDMR (red)

467 sequencing datasets. (C) Computed ancestry in a pie chart. Ancestry is classified in the following

468 categories: European (EUR-Red), African (AFR-Blue), American (AMR-Purple), Mixed (MIX-

469 Orange), East Asian (EAS-Light Purple), South Asian (SAS-Yellow). (D) Major portal updates in

470 a timeline starting in September of 2019 through March 2021.

\section{Figure 2. PDXNet sequencing data page on the PDXNet Portal}

472 Components of the PDXNet sequencing data page. (A) Panel shows summary statistics including 473 number of sequencing files (green), contributors (yellow), total samples (orange), and total patients 474 (blue). Also, shown are donut plots for contributors, sample types, disease type, experimental strategy, WES contributors, and RNA-Seq contributors. (B) Panel shows metadata for the PDXNet sequencing data in a spreadsheet format. The interface supports searching and sorting metadata. Users can copy, print, and download metadata into accessible formats.

Figure 3. Examples figures generated from the HRD-TMB-MSI page on the PDXNet Portal

Plots generated on the PDXNet Portal HRD-TMB-MSI page (A) Plot of Homologous Recombination Deficiency (HRD) computed from sequencing data provided by PDXNet researchers. The plot shows HRD by disease type (B) Plot of Tumor Mutational Burden (TMB) computed from sequencing data provided by PDXNet researchers. The plot shows TMB by disease type. (C) Plot of TMB computed from sequencing data provided by PDXNet researchers, by disease type.

\section{Figure 4. Tumor volume data page on the PDXNet Portal}

Components of the PDXNet tumor volume page. The figure shows a filtered dataset. (A) Panel shows summary statistics including number tumor volume datasets (green), number of models in the selected dataset (yellow), total number of patients (blue), and total number of diseases represented (red). (B) Panel shows the tumor volume data organized by the treatment arm. The user can control plot level (animal or treatment arm) and plot pattern (multiple or combined) 
PDXNet Portal

\section{2}

493

494

495

496

497

498

499

500

501

502

503

504

505

506

507

508

509

510

511

512

513

514

515

516

517

518

519

520

521

522

523

524

525

526

527

528

529

530

\section{Supplementary Materials}

\section{PDXNet Consortium Members}

Matthew Bailey 1, Emilio Cortes-Sanchez 1, Lacey E. Dobrolecki 1, Sandra Scherer 1, ChiehHsiang Yang 1, Maihi Fujita 1, Zhengtao Chu 1, Ling Zhao 1, Andrew Butterfield 1, Argun Akcakanat $^{2}$, Gao Boning ${ }^{2}$, Kurt Evans ${ }^{2}$, Bingliang Fang ${ }^{2}$, Don Gibbons ${ }^{2}$, Vanessa Jensen ${ }^{2}$, Dara Keener-Admin ${ }^{2}$, Michael Kim ${ }^{2}$, Scott Kopetz ${ }^{2}$, Mourad Majidi ${ }^{2}$, David Menter ${ }^{2}$, John Minna $^{2}$, Hyunsil Park ${ }^{2}$, Fei Yang ${ }^{2}$, Brenda Timmons ${ }^{2}$, Jing Wang ${ }^{2}$, Shannon Westin ${ }^{2}$, Timothy Yap $^{2}$, Jianhua Zhang ${ }^{2}$, Ran Zhang ${ }^{2}$, Min Jin Ha ${ }^{2}$, Huiqin Chen ${ }^{2}$, Yuanxin $\mathrm{Xi}^{2}$, Luc Girard ${ }^{2}$, Erkan Yucan ${ }^{2}$, Bryce P Kirby ${ }^{2}$, Bingbing Dai ${ }^{2}$, Yi Xu ${ }^{2}$, Alexey Sorokin ${ }^{2}$, Kelly Gale ${ }^{2}$, Jithesh Augustine ${ }^{2}$, Stephen Scott ${ }^{2}$, Ismail Meraz ${ }^{2}$, Dylan Fingerman ${ }^{3}$, Andrew Kossenkov ${ }^{3}$, Qin Liu ${ }^{3}$, Min Xiao ${ }^{3}$, Jayamanna Wickramasinghe ${ }^{3}$, Haiyin Lin ${ }^{3}$, Eric Ramirez-Salazar ${ }^{3}$, Kate Nathanson ${ }^{4}$, Mike Tetzlaff ${ }^{2}$, George Xu ${ }^{5}$, Vashisht G. Yennu-Nanda ${ }^{6}$, Rebecca Aft ${ }^{7}$, Jessica Andrews ${ }^{7}$, Alicia Asaro ${ }^{7}$, Song Cao ${ }^{7}$, Feng Chen ${ }^{7}$, Sherri Davies ${ }^{7}$, John DiPersio ${ }^{7}$, Ryan Fields ${ }^{7}$, Steven Foltz $^{7}$, Katherine Fuh ${ }^{7}$, Kian Lim ${ }^{7}$, Jason Held ${ }^{7}$, Jeremy Hoog ${ }^{7}$, Reyka G. Jayasinghe ${ }^{7}$, Yize Li ${ }^{7}$, Jinqin Luo ${ }^{7}$, Cynthia Ma ${ }^{7}$, Jay Mashl ${ }^{7}$, Chia-Kuei Mo ${ }^{7}$, Fernanda Rodriguez ${ }^{7}$, Hua Sun ${ }^{7}$, Nadezhda V. Terekhanova ${ }^{7}$, Rose Tipton ${ }^{7}$, Brian VanTine ${ }^{7}$, Andrea Wang-Gillam ${ }^{7}$, Mike Wendl ${ }^{7}$, Yige $\mathrm{Wu}^{7}$, Matt Wyczalkowski ${ }^{7}$, Lijun Yao ${ }^{7}$, Daniel Cui Zhou ${ }^{7}$, Matthew Ellis ${ }^{8}$, Michael Ittmann ${ }^{8}$, Susan Hilsenbeck ${ }^{8}$, Bert O'Malley ${ }^{8}$, Amanda Kirane ${ }^{9}$, May Cho ${ }^{9}$, David Gandara ${ }^{9}$, Jonathan Reiss ${ }^{9}$, Tiffany Le ${ }^{9}$, Ralph De Vere White ${ }^{9}$, Cliff Tepper ${ }^{9}$, David Cooke ${ }^{9}$, Luis Godoy ${ }^{9}$, Lisa Brown ${ }^{9}$, Marc Dall'Era ${ }^{9}$, Christopher Evans ${ }^{9}$, Rashmi Verma ${ }^{9}$, Sepideh Gholami ${ }^{9}$, David J Segal ${ }^{9}$, John Albeck ${ }^{9}$, Edward Pugh ${ }^{9}$, Susan Stewart ${ }^{9}$, David Rocke ${ }^{9}$, Hongyong Zhang ${ }^{10}$, Nicole Coggins ${ }^{10}$, Ana Estrada ${ }^{10}$, Ted Toal ${ }^{10}$, Alexa Morales ${ }^{10}$, Guadalupe Polanco Echeverry ${ }^{10}$, Sienna Rocha ${ }^{10}$, Ai-Hong Ma ${ }^{9,11}$

1: Huntsman Cancer Institute, Salt Lake City, UT.

2: The University of Texas M.D. Anderson Cancer Center, Houston, TX.

3: The Wistar Institute, Philadelphia, PA.

4: Abramson Cancer Center, The University of Pennsylvania, Philadelphia, PA, USA

5: Hospital of the University of Pennsylvania, Philadelphia, PA, USA

6: Department of Melanoma Medical Oncology, MD Anderson Cancer Center, Houston, TX.

7: Washington University School of Medicine, St. Louis, MO.

8: Baylor College of Medicine, Houston, TX.

9: UC Davis Comprehensive Cancer Center, University of California - Davis, Davis, CA.

10: UC Davis Comprehensive Genomics Center, University of California - Davis, Davis, CA.

11: Jamaica Plains VA Medical Center, Veteran Administration, Jamaica Plains, MA. 
PDXNet Portal

\section{PDXNet Member Contribution}

PDXNet Leadership and Data Submission

AW, BDD, BW, CJB, CXP, DAD, FMB, JD, JM, JHC, JR, GW, LCC, LD, MD, MH, MSC, MTW, NM, PNR, SK, SL, TW, YAE

\section{Portal Development}

Portal Integration Planning

\section{Writing Manuscript}

544 SK, MWL, DAD, JG, JHC 
PDXNet Portal

\section{Supplementary Tables}

548 Supplementary Table 1. Metadata associated with Hematoxylin and eosin (H\&E) Images

549 on the PDXNet Portal.

\section{Hematoxylin and Eosin (H\&E) Image Metadata}

\begin{tabular}{|c|c|}
\hline Age & Percent stromal content \\
\hline Cell annotation available? & Percent tumor content \\
\hline Contributor & Primary cancer site \\
\hline CTEP Code & Proteomics \\
\hline Diagnosis Subtype & Race \\
\hline Disease Type & Regional annotation available? \\
\hline Engraftment site & RNA-Seq/ Exp array \\
\hline Ethnicity & Sample ID \\
\hline Gender & Sample Type \\
\hline Histology & SNP array \\
\hline Image file name & Stain \\
\hline Is information of this model already in CGC? & Staining/scanning method available? \\
\hline Magnification & Thumbnail \\
\hline Metastatic site & Treatment \\
\hline Model ID & Treatment information in patient tumor \\
\hline Mouse strain & Treatment information in PDX tumor \\
\hline Note & Tumor Biomarkers \\
\hline Original Species & Tumor differentiation \\
\hline Other pathology notes & Tumor Grade \\
\hline Passage & Tumor Stage \\
\hline Patient ID & WES/Mutations \\
\hline Percent necrotic content & \\
\hline
\end{tabular}


PDXNet Portal

552 Supplementary Table 2. Supportive interactive table options on the PDXNet portal

\begin{tabular}{l} 
Supported Interactive \\
Tables and Charts \\
\hline Area Chart \\
Col Heatmap \\
Horizontal Bar Chart \\
Horizontal Stacked Bar Chart \\
Line Chart \\
Row Heatmap \\
Scatter Chart \\
Table \\
Table Bar Chart \\
Treemap
\end{tabular}

Supplementary Table 3. Data summary options available through interactive tables on the 558 PDXNet Portal

\begin{tabular}{ll}
\hline \multicolumn{2}{c}{ Interactive Table Data Summary Options } \\
\hline Average & Median \\
Count & Minimum \\
Count as fraction of column & Sample variance \\
Count as fraction of row & Standard deviation \\
Count as fraction of total & Sum \\
Count unique values & Sum as fraction of column \\
First & Sum as fraction of row \\
Integer sum & sum as fraction of total \\
Last & sum over sum \\
List unique values & $80 \%$ lower bound \\
maximum & $80 \%$ upper bound \\
\hline
\end{tabular}


PDXNet Portal

561 Supplementary Table 4. PDXNet metadata fields available on the Interactive Table 562 Explorer on the PDXNet Portal

\begin{tabular}{|c|c|}
\hline \multicolumn{2}{|c|}{$\begin{array}{l}\text { PDXNet Metadata Fields Available on the } \\
\text { Interactive Table Explorer }\end{array}$} \\
\hline Access Level & File size \\
\hline Availability & Gender \\
\hline Capture Assembly & Investigations \\
\hline Capture Kit & Is FFPE \\
\hline Case id & Model id \\
\hline Comments & Paired End \\
\hline Contributor & Patient id \\
\hline Created Date and time & Platform \\
\hline Data Category & Public \\
\hline Data Format & Sample ID \\
\hline Data Type & Sample type \\
\hline experimental strategy & Tumor id \\
\hline File name & \\
\hline
\end{tabular}

563

564 
bioRxiv preprint doi: https://doi.org/10.1101/2021.1015.464537. this version posted October 28,2021 . The copyright holder for this preprint (which was not certified by peer review) is the author/funder, who has granted bioRxiv a license to display the preprint in perpetuity. It is made available under aCC-BY-NC-ND 4.0 International license.

PDXNet Portal

565 Supplementary Table 5. Patient-derived model repository sequencing data processed with 566 standardized PDXNet workflows referenced on the PDXNet Portal

\begin{tabular}{lccc}
\hline & Overall & RNA-Seq & WES \\
Sample Type, $\mathbf{n}(\%)$ & $\mathrm{N}=9492$ & $\mathrm{~N}=4480$ & $\mathrm{~N}=5012$ \\
\hline Normal & $520(5.5 \%)$ & $0(0 \%)$ & $520(10 \%)$ \\
Organoid & $304(3.2 \%)$ & $152(3.4 \%)$ & $152(3.0 \%)$ \\
PDC & $276(2.8 \%)$ & $124(2.8 \%)$ & $152(3.0)$ \\
PDX & $7846(83 \%)$ & $3930(88 \%)$ & $3916(78 \%)$ \\
Tumor & $542(5.7)$ & $274(6.1 \%)$ & $268(5.3 \%)$ \\
unknown & $4(<0.1 \%)$ & $0(0 \%)$ & $4(<0.1 \%)$ \\
\hline
\end{tabular}


PDXNet Portal

568 Supplementary Table 6. Standardized PDXNet bioinformatics workflows linked to the 569 PDXNet Portal

\section{Workflow Description}

\section{RNA-Seq}

Prepare Multi-sample Data

PDX RNA Expression Estimation Workflow

PDX RNA Expression Estimation Workflow (Single End)

RNA Expression Estimation Workflow Patient Tumor

RNA Expression Workflow Patient Tumor (Single End)

Whole Exome Sequence

PDX WES CNV (Xenome) Tumor-Normal Workflows

PDX WES Tumor-Normal (Xenome) with Variant Calling, CNV Estimation, TMB, $\mathrm{MSI}$, and HRD Scores

PDX WES Tumor-Only (Xenome) with Variant Calling, MSI, and TMB Scores

WES Tumor-Normal with Variant Calling, CNV Estimation, TMB, MSI, and HRD

Scores

WES Tumor-Only with Variant Calling, MSI, and TMB Scores

WES Tumor-Only from BAM (Variant, MSI, TMB)

WES Tumor-Normal from BAM(Variant, CNV, HRD, MSI, TMB)

\section{SNP Array}

SNP Array Tumor-Only Workflow for Illumina Infinium Omni 2.5 Exome-8 (Version

1.4) Snp Array

\section{Quality}

PDX WES Sample QC

PDX Sample QC 
PDXNet Portal

572 Supplementary Table 7. Summary of computed ancestry for PDXNet Models

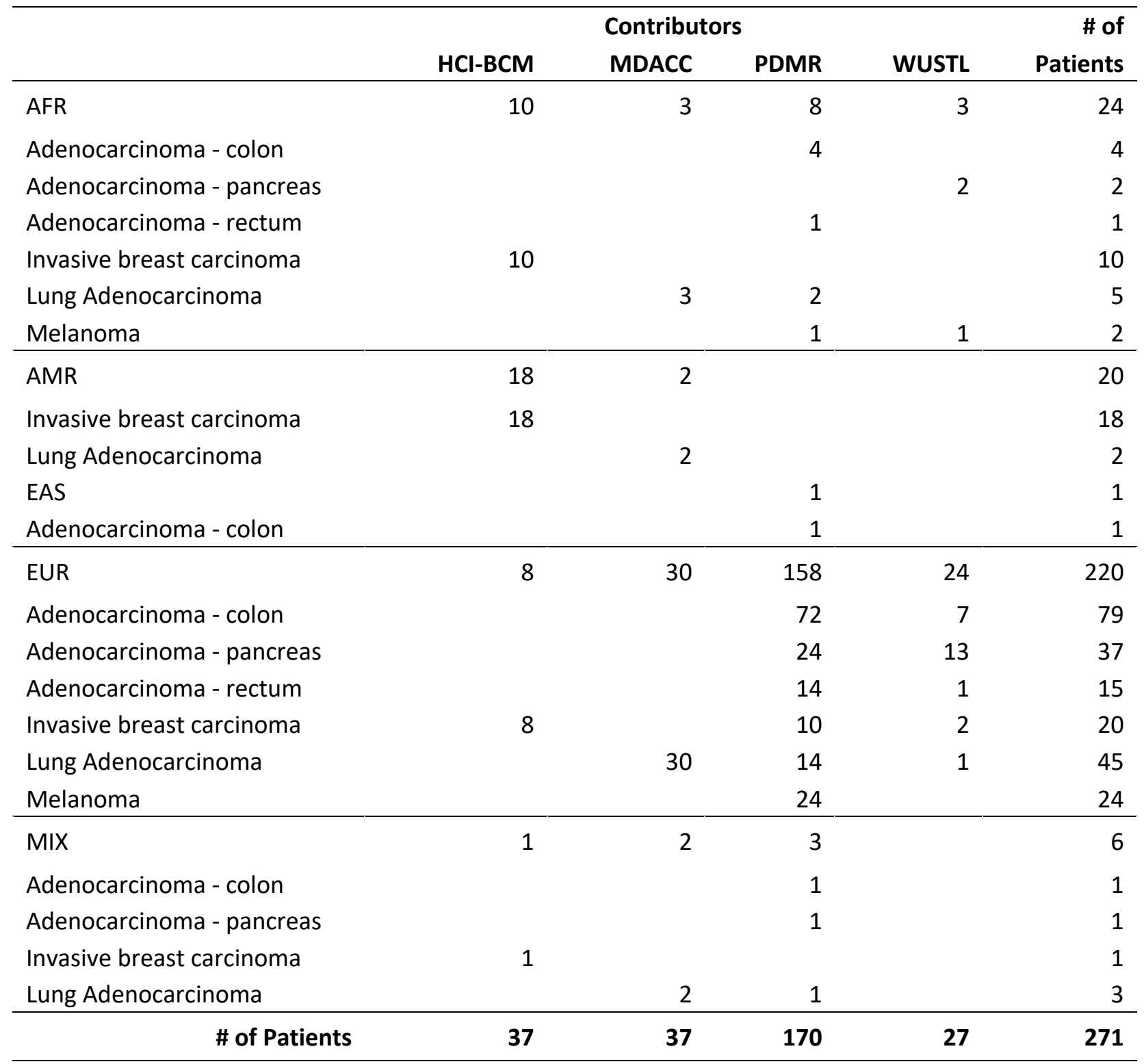

HCI-BCM: Huntsman Cancer Center, MDACC: MD Anderson Cancer Center, PDMR: Patient-Derived Model Repository, WUSTL: Washington University at St. Louis 
PDXNet Portal

575 Supplementary Table 8. Summary of PDMR tumor volume dataset on the PDXNet portal 576 shown by disease type and treatment

\begin{tabular}{|c|c|c|c|c|c|c|c|c|c|}
\hline \multirow[b]{2}{*}{ Disease } & \multicolumn{8}{|c|}{ Treatment } & \multirow[b]{2}{*}{ Total } \\
\hline & Control & $5-\mathrm{FU}$ & Carboplatin & Erlotinib & Gemcitabine & Irinotecan & Paclitaxel & Vemurafenib & \\
\hline Colon & 13 & 10 & 10 & 7 & 11 & 11 & 11 & 7 & 81 \\
\hline Lung & 13 & 9 & 10 & 8 & 10 & 10 & 10 & 8 & 78 \\
\hline Pancreas & 12 & 11 & 1 & 9 & 11 & 11 & 11 & 7 & 82 \\
\hline Skin & 9 & 7 & 1 & 3 & 7 & 6 & 7 & 3 & 48 \\
\hline Bladder & 7 & 5 & 1 & 3 & 5 & 6 & 6 & 4 & 42 \\
\hline Head and Neck & 10 & 5 & 1 & 4 & 6 & 6 & 6 & 3 & 45 \\
\hline Kidney & 2 & 2 & & 1 & 2 & 2 & 2 & 1 & 14 \\
\hline Ovarian & 1 & 1 & 1 & & 1 & 1 & 1 & & 6 \\
\hline Uterine & 4 & 4 & 1 & 1 & 4 & 4 & 4 & 1 & 26 \\
\hline Gastric & 1 & 1 & 1 & 1 & 1 & 1 & 1 & 1 & 8 \\
\hline $\begin{array}{l}\text { Soft Tissue } \\
\text { Neoplasm }\end{array}$ & 9 & 8 & 3 & 2 & 9 & 9 & 9 & 1 & 54 \\
\hline Endocrine & 1 & 1 & 3 & 1 & 1 & 1 & 1 & & 7 \\
\hline Bone & 1 & 1 & 1 & & 1 & & 1 & & 5 \\
\hline CNS & 3 & 3 & 1 & 2 & 3 & 3 & 3 & 2 & 22 \\
\hline Rectum & 1 & 1 & 1 & & 1 & 1 & 1 & & 6 \\
\hline Grand Total & 87 & 69 & 36 & 42 & 73 & 72 & 74 & 38 & 524 \\
\hline
\end{tabular}


PDXNet Portal

\section{Graphical User Interface Screenshots}

580 Resources

581

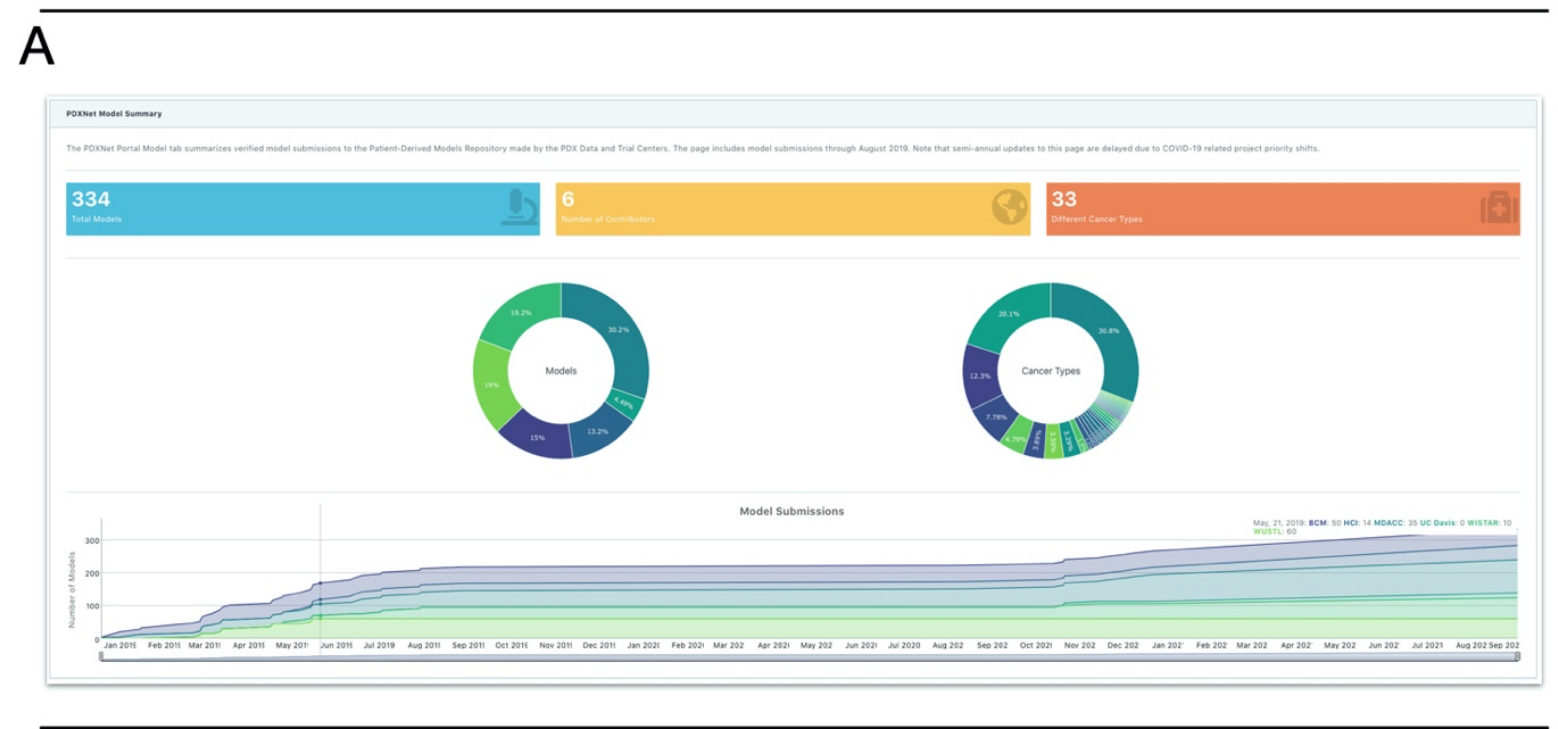

B

\section{Supplement Figure 1. PDX models generated by PDXNet researchers shown on the PDXNet}

\section{Portal}

Figure shows components of the PDXNet Model sequencing data page in separate panels (A) Panel shows summary statistics including number of total models (blue), number of contributors (yellow), and number of cancer types (orange). Also, shown are donut plots for contributors and cancer types. Below the donut plots is a chart showing the number of models generated since January 2019. (B) Panel shows metadata for the PDXNet PDX models in a spreadsheet format. The interface supports searching and sorting metadata. Users can copy, print, and download metadata into accessible formats. 
bioRxiv preprint doi: https://doi.org/10.1101/2021.10.15.464537; this version posted October 28, 2021. The copyright holder for this preprint (which was not certified by peer review) is the author/funder, who has granted bioRxiv a license to display the preprint in perpetuity. It is made available under aCC-BY-NC-ND 4.0 International license.

PDXNet Portal

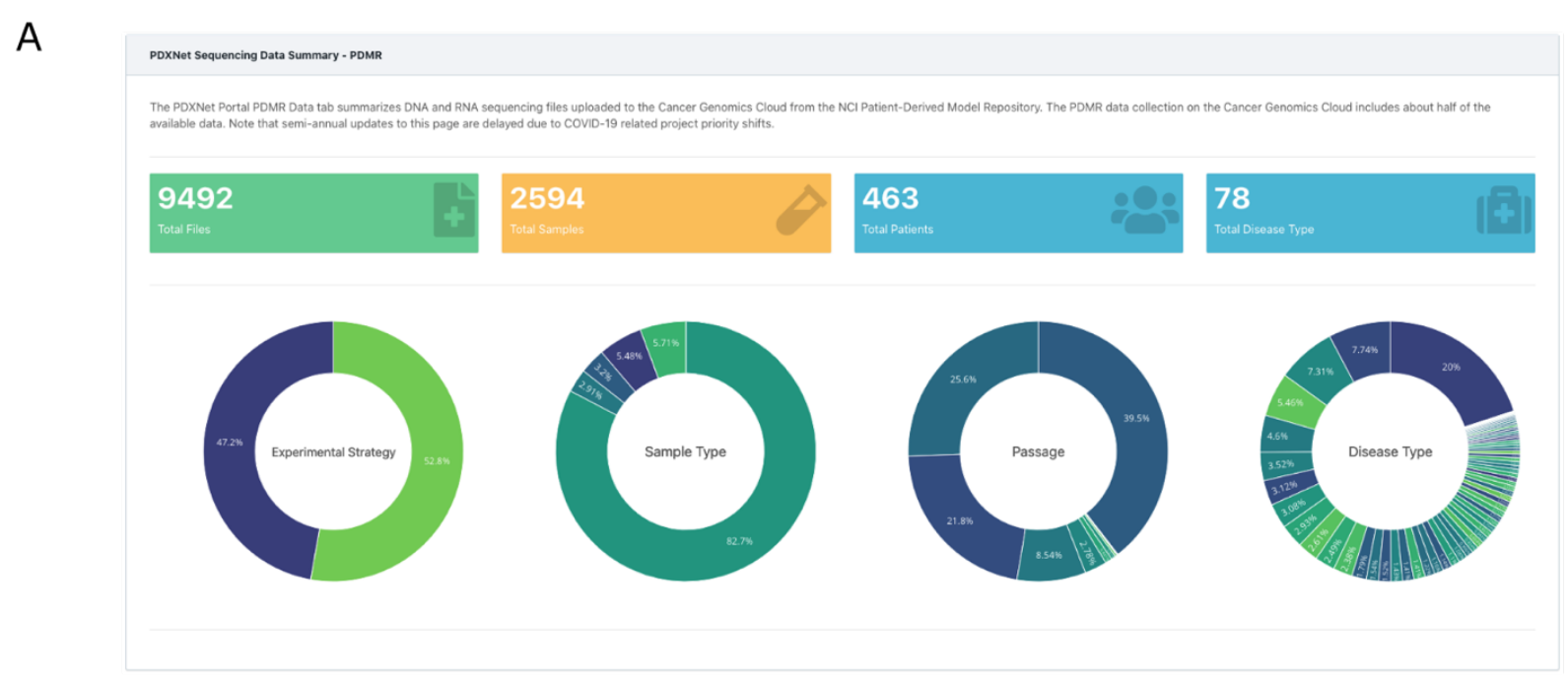

B

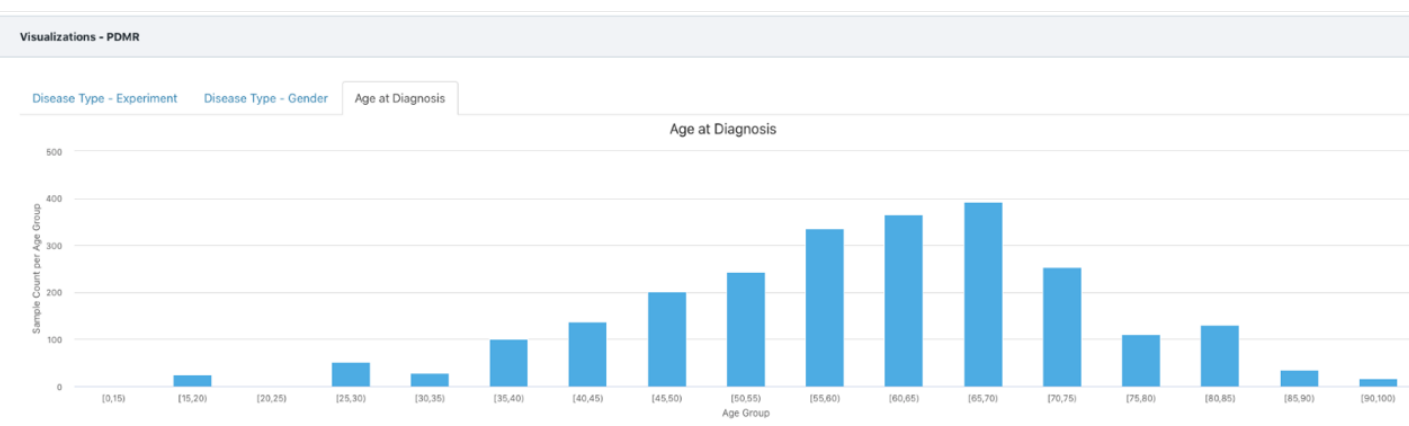

C

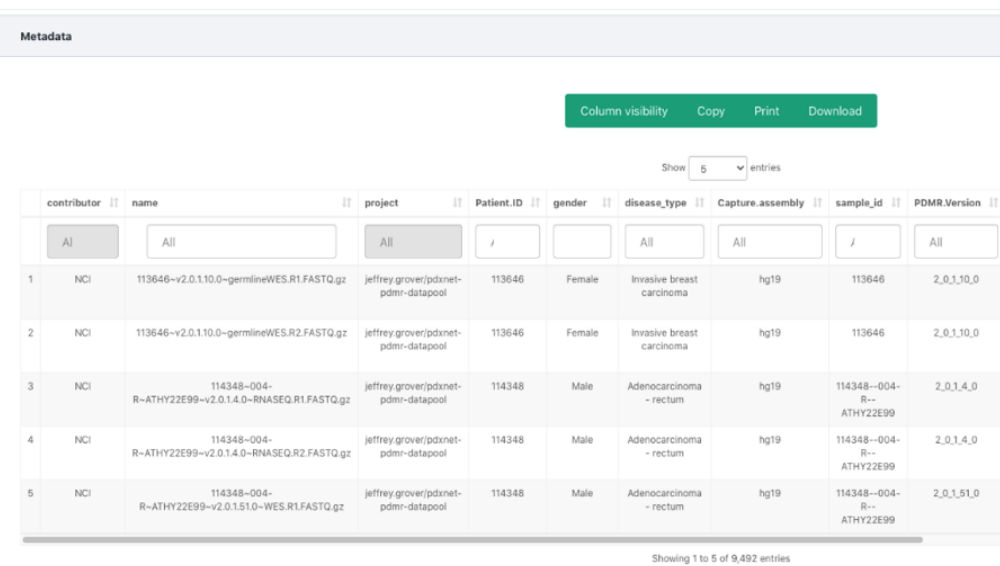

Showing 105 of 9 , 992 entities 
PDXNet Portal

596 Figure shows components of the PDMR sequencing data page in separate panels (A) Panel shows 597 summary statistics including number of sequencing files (green), contributors (yellow), total 598 samples (orange), and total patients (blue). Also, shown are donut plots for contributors, sample 599 types, disease type, experimental strategy, WES contributors, and RNA-Seq contributors. (B) 600 Panel shows age from PDMR patients on a bar chart. (C) Panel shows metadata for the PDMR 601 sequencing data in a spreadsheet format. The interface supports searching and sorting metadata. 602 Users can copy, print, and download metadata into accessible formats.

603 
bioRxiv preprint doi: https://doi.org/10.1101/2021.10.15.464537; this version posted October 28, 2021. The copyright holder for this preprint (which was not certified by peer review) is the author/funder, who has granted bioRxiv a license to display the preprint in perpetuity. It is made available under aCC-BY-NC-ND 4.0 International license.

PDXNet Portal

604

A

Image - Summary

\section{4}

Total Images

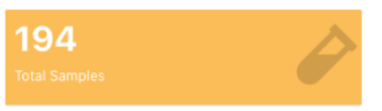

\section{7}

Total Patients

\section{4}

\section{Total D}

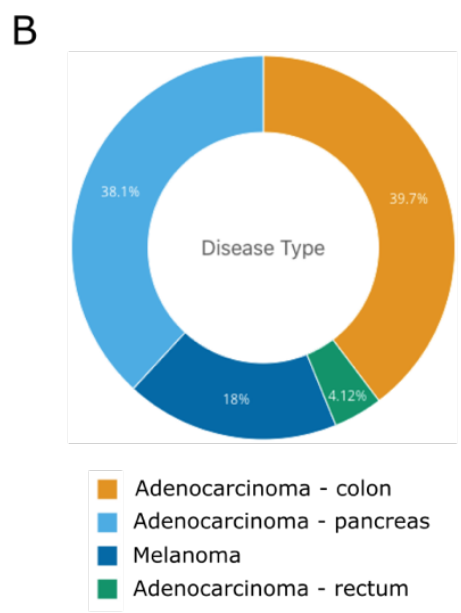

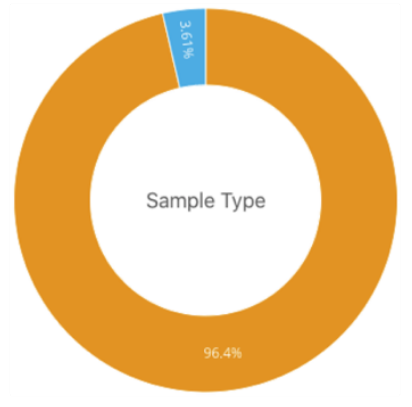

PDX

Primary Tumor

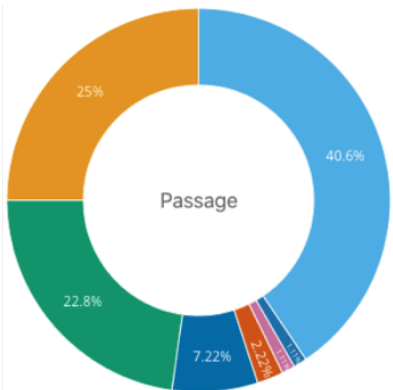

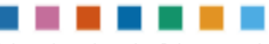

C

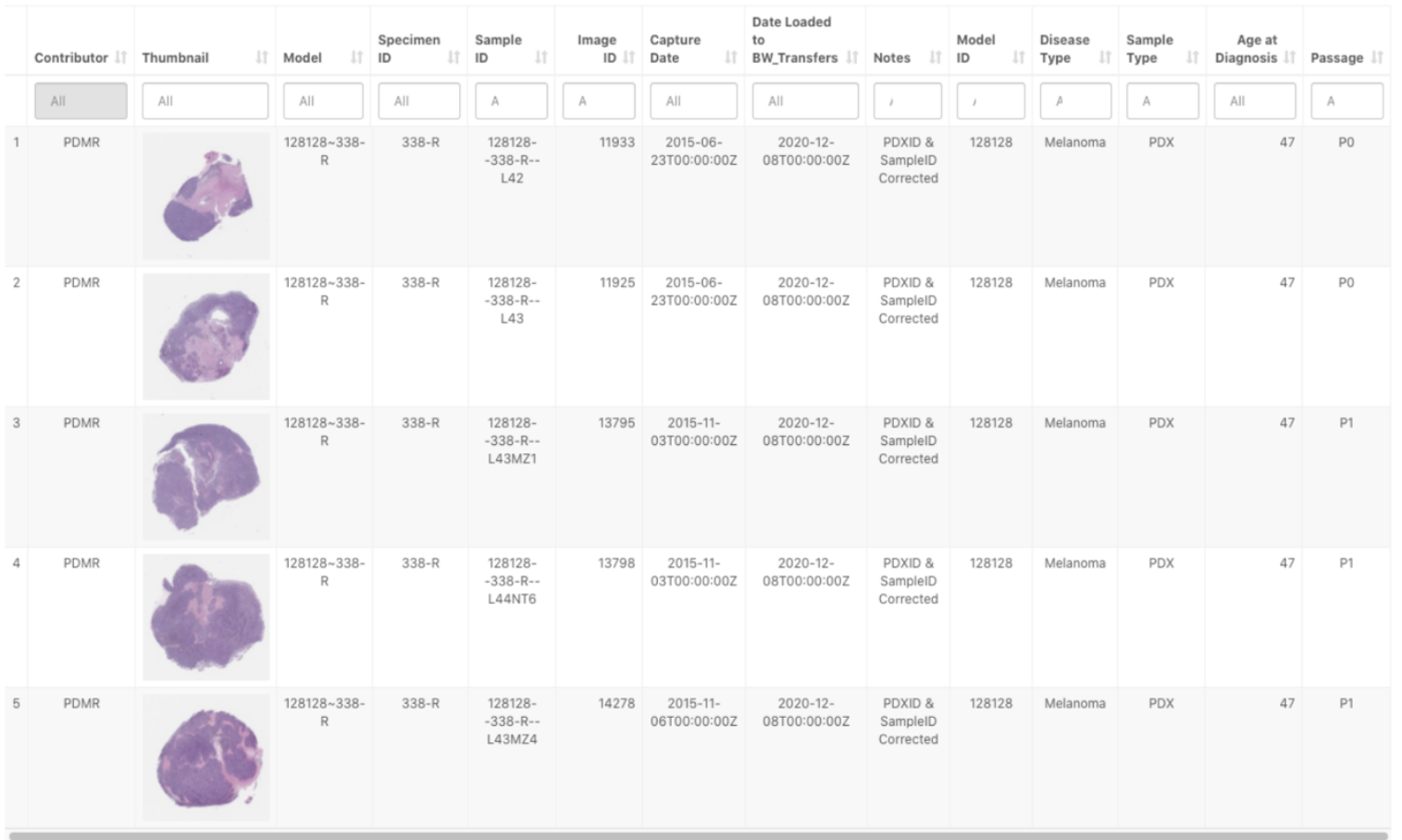

Showing 1 to 5 of 194 entries 
PDXNet Portal

608 Figure shows components of the PDMR image data page in separate panels (A) Panel shows 609 summary statistics including number of images (green), contributors (yellow), total patients (blue), 610 and total disease types (red). (B) Panel B shows donut plots for disease type, sample type, and 611 passage. (C) Panel shows metadata for the PDMR image data in a spreadsheet format. The 612 interface supports searching and sorting metadata. Users can copy, print, and download metadata 613 into accessible formats. 
bioRxiv preprint doi: https://doi.org/10.1101/2021.10.15.464537; this version posted October 28, 2021. The copyright holder for this preprint (which was not certified by peer review) is the author/funder, who has granted bioRxiv a license to display the preprint in perpetuity. It is made available under aCC-BY-NC-ND 4.0 International license.

PDXNet Portal

614

Interactive Exploration

The PDXNet Portal Interactive tab allows users to interactviely create tables from the list of DNA and RNA sequencing file uploaded to the Cancer

Genomics Cloud by the PDX Data and Trial Centers. The page includes data uploads through August 2019.

Pivot Tables

PDMR Files $\quad$ PDTC Files

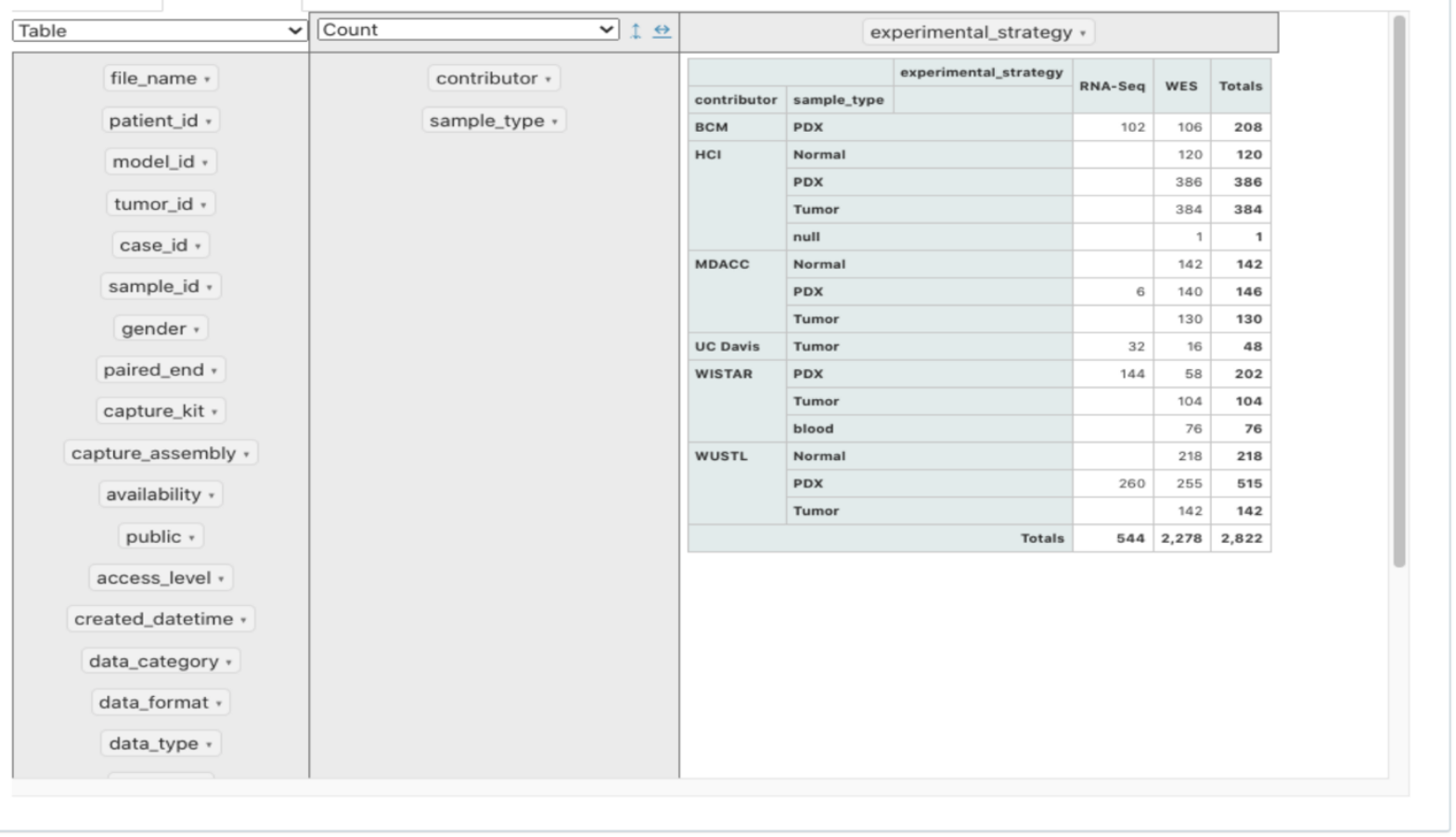

\section{Supplement Figure 4. Interactive data explorer page on the PDXNet Portal}

617 Figure shows the interactive exploration page on the PDXNet portal. The user can interactively 618 create a pivot table with either the metadata from the PDXNet sequencing data or the PDMR sequencing data. Constructing the table involves dragging and dropping table fields on the left side to the table area (green) on the right side of the screen. 
bioRxiv preprint doi: https://doi.org/10.1101/2021.10.15.464537; this version posted October 28, 2021. The copyright holder for this preprint (which was not certified by peer review) is the author/funder, who has granted bioRxiv a license to display the preprint in perpetuity. It is made available under aCC-BY-NC-ND 4.0 International license.

PDXNet Portal

625 Figure shows a section of the workflow page on the PDXNet portal. The page includes brief

O PDX WES CNV (Xenome) Tumor-Normal Worktlow
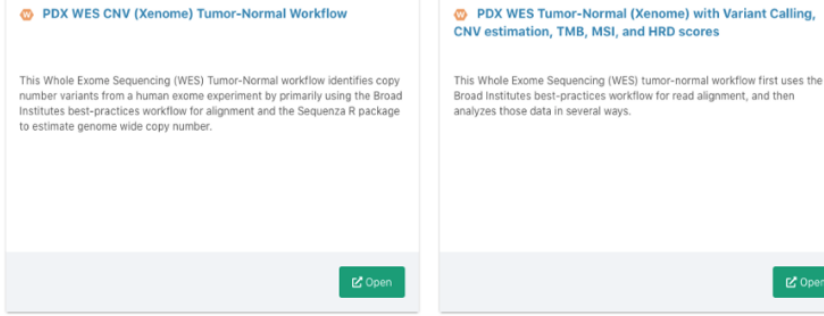

W. WES Tumor-Normal with
TMB, MSI, and HRD scores
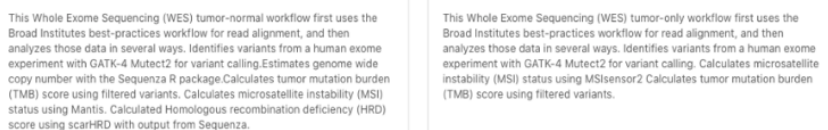

ccore using scartipg with outtout trom Sequenza

\section{Portal} extent array data. The page includes links to comprehensive workflow documentation on the Cancer Genomics Cloud Public Apps Gallery; where the workflows are made publicly available. 
PDXNet Portal

A

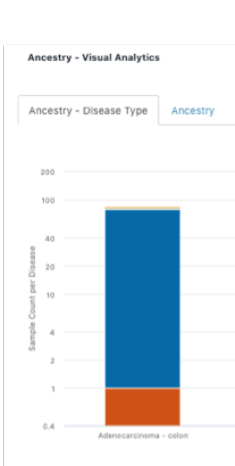

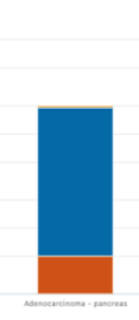
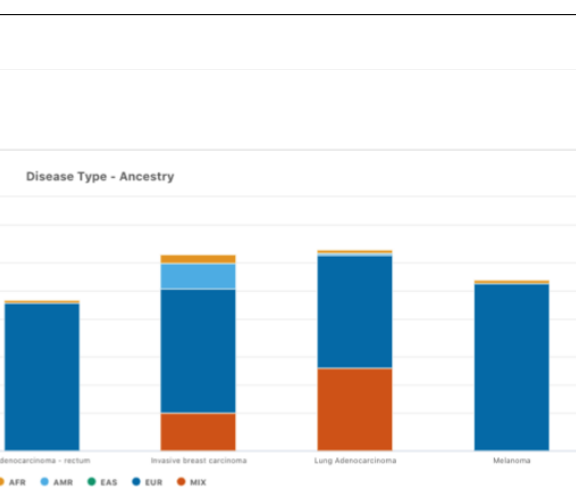

B

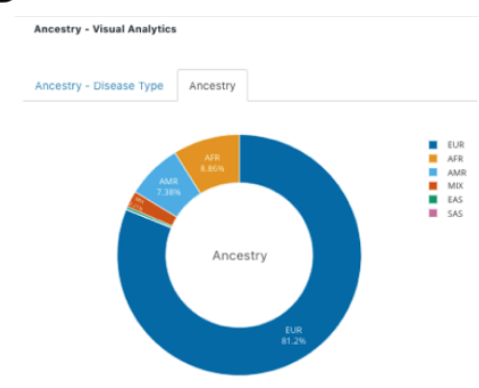

C

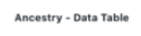
PDXNet Portal

Figure shows plots generated on the ancestry data page of the PDXNet portal (A) Panel A shows a stacked bar chart with each bar corresponding to a user selected disease. Each bar shows the ancestry composition of available samples by color. The ancestry algorithm classifies samples as African (AFR), American (AMR), East Asian (EAS), South Asian (SAS), and Mixed (MIX). (B) Panel shows computed ancestry in a pie chart. Ancestry is classified in the following categories: European(EUR), African(AFR), American(AMR), Mixed(MIX), East Asian(EAS), South Asian(SAS). (C) Panel shows ancestry metadata for the processed sequencing data in a spreadsheet format. The interface supports searching and sorting metadata. Users can copy, print, and download metadata into accessible formats. 
bioRxiv preprint doi: https://doi.org/10.1101/2021.10.15.464537; this version posted October 28, 2021. The copyright holder for this preprint (which was not certified by peer review) is the author/funder, who has granted bioRxiv a license to display the preprint in perpetuity. It is made available under aCC-BY-NC-ND 4.0 International license.

PDXNet Portal

A
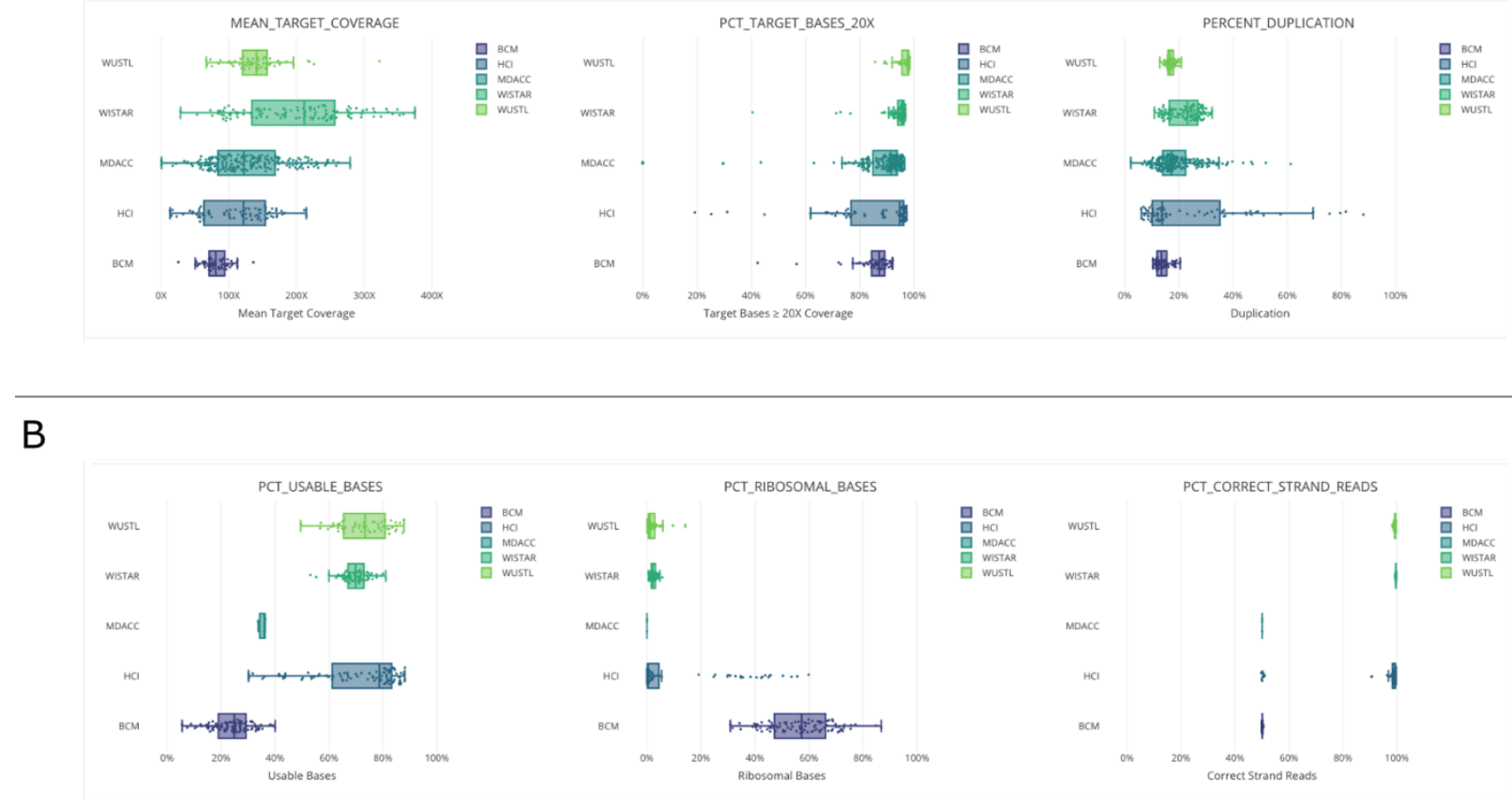

C

MEAN_TARGET_COVERAGE

PCT_TARGET_BASES_20X

PERCENT_DUPLICATION
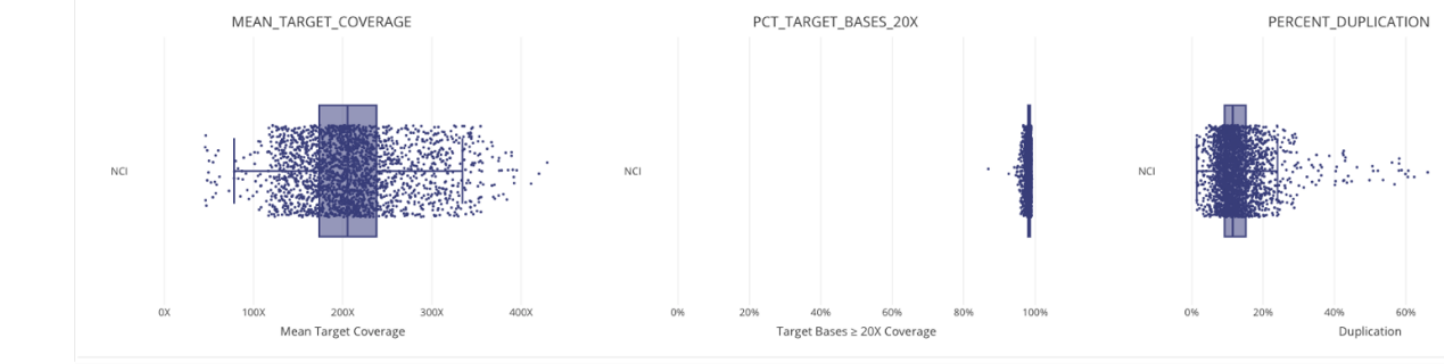

D

PCT_USABLE_BASES

PCT_RIBOSOMAL_BASES

PCT_CORRECT_STRAND_READS
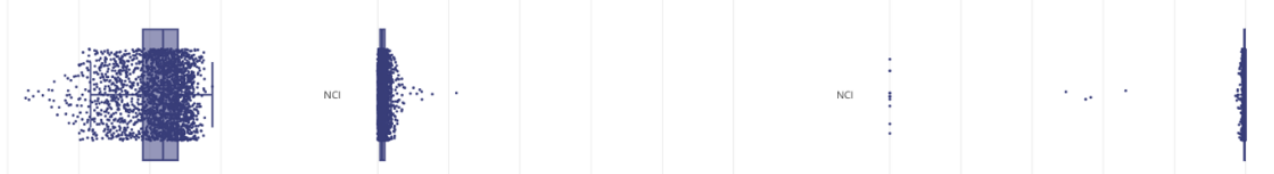

643 Supplement Figure 7. Sequencing quality control plots examples generated on the PDXNet 644 Portal

645 Figure shows sequencing data QC figures generated on the quality control page of the PDXNet 646 
PDXNet Portal

647 as box plots with data for each PDX Development and Trial Center presented as a different box 648 plot. (B) Plot shows percent usable basis, percent ribosomal basis, and percent correct strand reads 649 as box plots with data for each PDX Development and Trial Center presented as a different box 650 plot. (C) Plot shows mean target coverage, percent target coverage at 20x, and percent duplication 651 as box plots generated from PDMR data. (D) Plot shows percent usable basis, percent ribosomal 652 basis, and percent correct strand reads as box plots generated from PDMR data. 
bioRxiv preprint doi: https://doi.org/10.1101/2021.10.15.464537; this version posted October 28, 2021. The copyright holder for this preprint (which was not certified by peer review) is the author/funder, who has granted bioRxiv a license to display the preprint in perpetuity. It is made available under aCC-BY-NC-ND 4.0 International license.

PDXNet Portal 